\title{
Subchronic inhalation toxicity of gold nanoparticles
}

\author{
Jae Hyuck Sung ${ }^{1}$, Jun Ho Ji ${ }^{2}$, Jung Duck Park ${ }^{3}$, Moon Yong Song ${ }^{1}$, Kyung Seuk Song ${ }^{1}$, Hyeon Ryol Ryu', \\ Jin Uk Yoon ${ }^{4}$, Ki Soo Jeon ${ }^{4}$, Jayoung Jeong ${ }^{5}$, Beom Seok Han', Yong Hyun Chung ${ }^{6}$, Hee Kyung Chang ${ }^{7}$, \\ Ji Hyun Lee ${ }^{9}$, Dong Won Kim³, Bruce J Kelman ${ }^{8}$ and II Je Yu ${ }^{9^{*}}$
}

\begin{abstract}
Background: Gold nanoparticles are widely used in consumer products, including cosmetics, food packaging, beverages, toothpaste, automobiles, and lubricants. With this increase in consumer products containing gold nanoparticles, the potential for worker exposure to gold nanoparticles will also increase. Only a few studies have produced data on the in vivo toxicology of gold nanoparticles, meaning that the absorption, distribution, metabolism, and excretion (ADME) of gold nanoparticles remain unclear.

Results: The toxicity of gold nanoparticles was studied in Sprague Dawley rats by inhalation. Seven-week-old rats, weighing approximately $200 \mathrm{~g}$ (males) and $145 \mathrm{~g}$ (females), were divided into 4 groups (10 rats in each group): fresh-air control, low-dose $\left(2.36 \times 10^{4}\right.$ particle $\left./ \mathrm{cm}^{3}, 0.04 \mathrm{\mu g} / \mathrm{m}^{3}\right)$, middle-dose $\left(2.36 \times 10^{5}\right.$ particle $\left./ \mathrm{cm}^{3}, 0.38 \mathrm{\mu g} / \mathrm{m}^{3}\right)$, and high-dose $\left(1.85 \times 10^{6}\right.$ particle/ $\left./ \mathrm{cm}^{3}, 20.02 \mu \mathrm{g} / \mathrm{m}^{3}\right)$. The animals were exposed to gold nanoparticles (average diameter 4-5 nm) for 6 hours/day, 5 days/week, for 90-days in a whole-body inhalation chamber. In addition to mortality and clinical observations, body weight, food consumption, and lung function were recorded weekly. At the end of the study, the rats were subjected to a full necropsy, blood samples were collected for hematology and clinical chemistry tests, and organ weights were measured. Cellular differential counts and cytotoxicity measurements, such as albumin, lactate dehydrogenase (LDH), and total protein were also monitored in a cellular bronchoalveolar lavage (BAL) fluid. Among lung function test measurements, tidal volume and minute volume showed a tendency to decrease comparing control and dose groups during the 90-days of exposure. Although no statistically significant differences were found in cellular differential counts, histopathologic examination showed minimal alveoli, an inflammatory infiltrate with a mixed cell type, and increased macrophages in the high-dose rats. Tissue distribution of gold nanoparticles showed a dose-dependent accumulation of gold in only lungs and kidneys with a gender-related difference in gold nanoparticles content in kidneys.

Conclusions: Lungs were the only organ in which there were dose-related changes in both male and female rats. Changes observed in lung histopathology and function in high-dose animals indicate that the highest concentration $\left(20 \mathrm{\mu g} / \mathrm{m}^{3}\right)$ is a LOAEL and the middle concentration $\left(0.38 \mathrm{\mu g} / \mathrm{m}^{3}\right)$ is a NOAEL for this study.
\end{abstract}

Keywords: gold nanoparticles, subchronic inhalation toxicity, tissue distribution, NOAEL

\section{Introduction}

Metallic gold $\left(\mathrm{Au}^{\circ}\right)$ is arguably considered to be the least corrosive and most biologically inert of all metals [1]. However, that does not mean $\mathrm{Au}^{\circ}$ has been shown to be completely inert in mammals. Human exposure to $\mathrm{Au}^{\circ}$ is common. Much of the earth's population has

\footnotetext{
* Correspondence: u1670916@chollian.net

${ }^{9}$ Toxicologcial Research Center, Hoseo University, Asan, Korea

Full list of author information is available at the end of the article
}

prolonged dermal contact to metallic gold in the form of jewelry and allergic contact dermatitis (ACD) has been documented as a result of exposure to dental restorations, gold jewelry, and use of gold in food [2-5]. The ingestion of gold-containing liquor beverages has resulted in allergic-type reactions similar to those seen after gold-allergic individuals are exposed to gold through medications or jewelry [3].

\section{Biomed Central}

(c) 2011 Sung et al; licensee BioMed Central Ltd. This is an Open Access article distributed under the terms of the Creative Commons Attribution License (http://creativecommons.org/licenses/by/2.0), which permits unrestricted use, distribution, and reproduction in any medium, provided the original work is properly cited. 
The presence of metallic gold has been visualized in human skin biopsies taken from areas of prolonged contact with the metal, such as rings and jewelry, confirming absorption of the solubilized metal even though intact stratum corneum. Skin samples taken from beneath the wedding bands of normal individuals ranged from 0.07 to $0.09 \mu \mathrm{g}$ of gold per gram of dry weight [6].

Metallic gold can be gradually dissolved by thiolcontaining molecules such as cysteine, penicillamine, and glutathione to yield gold (I) complexes [6]. It is possible that the more reactive $\mathrm{Au}^{+}$and $\mathrm{Au}^{3+}$ species could be a source of the toxicity attributed to $\mathrm{Au}^{\circ}$ [3]. Additionally, metallic gold used in jewelry and prostheses is ordinarily alloyed with other metals; even high-carat yellow gold contains minute quantities of copper and silver, while low-carat gold contains these metals plus zinc and nickel [1].

Gold nanoparticles are widely used in consumer products, including cosmetics, food packaging, beverages, toothpaste, automobiles, and air handling units. Among 1015 consumer nanotechnology-based products, nanogold-based products rank sixth out of 28 products [7]. In addition, many new nano-gold-based biomedical products are being developed for drug delivery, cancer therapy, diagnostic devices, and biosensing. With this increase in consumer products containing gold nanoparticles, the potential for worker exposure to gold nanoparticles will also increase, which has prompted (in part) a US NTP study of gold nanoparticles [8]. Only a few studies have produced data on the in vivo toxicology of gold nanoparticles, meaning that the absorption, distribution, metabolism, and excretion (ADME) of gold nanoparticles remain unclear. This is partially due to the high price of gold in long-term in vivo studies. The present subchronic 90-day inhalation toxicity study was designed to identify possible adverse effects and determine a LOAEL or NOAEL. The study was designed according to test guideline 413 from the Organization for Economic Cooperation and Development (OECD) [9], and to comply with Good Laboratory Practices (GLPs). In contrast to silver nanoparticles that are known to be ionized in biological media, gold nanoparticles are not normally ionized in biological media. Thus, inhalation exposure to gold nanoparticles provides the potential to separate the behavior of nanoparticles as particles from chemical effects.

\section{Materials and methods}

\section{Generation of gold nanoparticles}

Gold nanoparticles were generated as described in previous reports [10-13], and the rats exposed in a wholebody-type exposure chamber $\left(1.3 \mathrm{~m}^{3}\right.$, Dusturbo, Seoul). The generation system consisted of a small ceramic heater connected to an AC power supply and housed within a quartz tube case. The heater dimensions were $50 \times 5 \times 1.5 \mathrm{~mm}^{3}$, and a surface temperature of about $1500^{\circ} \mathrm{C}$ within a local heating area of $5 \times 10 \mathrm{~mm}^{2}$ was achieved in about 10 seconds [12]. For long-term testing, the source material (about $700 \mathrm{mg}$ of $24 \mathrm{ct}$ gold) was positioned at the highest temperature point. The quartz tube case was $70 \mathrm{~mm}$ in diameter and $200 \mathrm{~mm}$ long. Clean (dry and filtered) air was used as the carrier gas, and the gas flow maintained at $30.0 \mathrm{~L} / \mathrm{min}$ ( $\mathrm{Re}=572$, laminar flow regime) using a mass flow controller (MFC, AERA, FC-7810CD-4V, Japan) [10,11]. This generator has already been shown to generate nanoparticles from 1.8 to $6.1 \mathrm{~nm}$ (below $10 \mathrm{~nm}$ ) in diameter that do not agglomerate in air. XRD analysis using an X-ray diffractometer with $\mathrm{CuK} 2$ radiation previously showed that the particles generated are metallic gold, not gold oxides [13]. In the current study, the system produced different concentrations of nanoparticles (high, middle, and low) in three separate chambers. For the high-concentration chamber, the nanoparticle generator was operated at $45 \mathrm{~L} / \mathrm{min}$ (liters per minute) and mixed with $200 \mathrm{~L} / \mathrm{min}$ of clean ambient air. A portion of the high nanoparticle concentration was then diverted to the middle-concentration chamber using an MFC for the first dilution $(1.49 \pm 0.06 \mathrm{~L} / \mathrm{min}$, mean \pm S.E.), and a portion of the middle nanoparticle concentration then diverted to the low-concentration chamber using a second MFC $(14.94 \pm 0.02 \mathrm{~L} / \mathrm{min})$.

\section{Monitoring of inhalation chamber and analysis of gold nanoparticles}

In each chamber, nanoparticle distribution in terms of size was measured directly in real-time using a differential mobility analyzing system (DMAS); combining a differential mobility analyzer (Short type-DMA, 4220, HCT Co., Ltd. Korea, range 2-150 $\mathrm{nm}$ ) and condensation particle counter (CPC, 4312, HCT Co., Ltd. Korea, $0-10^{8} / \mathrm{cm}^{3}$ detection range). The nanoparticles were measured using sheath air at $5 \mathrm{~L} / \mathrm{min}$ and polydispersed aerosol air at $1 \mathrm{~L} / \mathrm{min}$ for the DMA and CPC, respectively.

The control chamber was supplied with HEPA-filtered fresh air. Particle concentration in the fresh-air control chamber was measured using a particle sensor (4123, HCT Co., Ltd. Korea) that consisted of two channels; 300-1000 $\mathrm{nm}$ and over $1000 \mathrm{~nm}$, to verify the performance of the HEPA filters. Previous studies have shown that the control chamber contains negligible nanoparticles if the HEPA filter is functioning correctly [14,15].

\section{Transmission Electron Microscopy}

The filters used to sample the fume particles were coated with carbon, mounted on an electron microscope grid (200 mesh, Veco, Eerbeek, Holland), and visualized under a field emission-transmission electron microscope 
(FE-TEM, JEM2100F, JEOL, JAPAN). The diameters of 800 randomly selected particles collected using a nanoparticle collector (nanoparticle collector, 4650, HCT Co., Ltd. Korea) were measured at a magnification of 100,000 , and the gold particles analyzed using an energy-dispersive $\mathrm{x}$-ray analyzer (EDX, TM200, Oxford, UK) at an accelerating voltage of $200 \mathrm{kV}$.

\section{Animals and Exposure Conditions}

Six-week-old male and female, specific-pathogen-free (SPF) Sprague-Dawley rats (Slc:SD) (originally derived from the Charles River SD in 1968) were purchased from SLC (Tokyo, Japan) and acclimated for 1 week before starting the experiments. During the acclimation and experimental periods, the rats were housed in polycarbonate cages (3 rats per cage) in a room with controlled temperature $\left(23 \pm 2^{\circ} \mathrm{C}\right)$, humidity $(55 \pm 7 \%)$, and a 12-h light/dark cycle. The rats were fed a rodent diet (Harlan Teklab, Plaster International Co., Seoul) and filtered water ad libitum. The 7-week-old rats, weighed approximately $200 \mathrm{~g}$ for the males and $145 \mathrm{~g}$ for the females at the beginning of exposure. The rats were divided into 4 groups (10 rats in each group/sex): freshair control, low-dose group (target dose, $2.5 \times 10^{4}$ particles $/ \mathrm{cm}^{3}, 1.0 \times 10^{6} \mathrm{~nm}^{2} / \mathrm{cm}^{3}$ surface area), middle-dose group (target dose, $2.5 \times 10^{5}$ particles $/ \mathrm{cm}^{3}, 6.0 \times 10^{6}$ $\mathrm{nm}^{2} / \mathrm{cm}^{3}$ surface area), and high-dose group (target dose, $1.2-2.8 \times 10^{6}$ particles $/ \mathrm{cm}^{3}, 3.0 \times 10^{8} \mathrm{~nm}^{2} / \mathrm{cm}^{3}$ surface area). The animals were exposed to the gold nanoparticles for 6 hours/day, 5 days/week, for 13-weeks, and housed in individual wire cages without food and water during the 6-hour exposure periods. The animals were examined daily on weekdays for any evidence of exposure-related effects, including respiratory, dermal, behavioral, nasal, or genitourinary changes suggestive of irritation. Body weights were evaluated at the time of purchase, at the time of grouping, after one day of exposure, once a week during exposure, and before necropsy. The experiment was approved by the KCL Institutional Animal Care and Use Committee.

\section{Biochemistry and Hematology}

At the conclusion of the 90-day experiment, the rats were 20 wks old. Before necropsy, food was withheld for $24 \mathrm{~h}$ and the rats were anesthetized with an overdose of sodium pentobarbital. Blood was then drawn from the abdominal aorta, collected in vacutainers, and analyzed for ALB (albumin), ALP (alkaline phosphatase), Ca (calcium), CHO (cholesterol), CRE (creatinine), gamma-GT (gamma-glutamyl transpeptidase), GLU (glucose), GOT (glutamic oxaloacetic transaminase), GPT (glutamic pyruvic transaminase), IP (inorganic phosphorus), LDH (lactate dehydrogenase), MG (magnesium), TP (total protein), UA (uric acid), BUN (blood urea nitrogen),
TBIL (total bilirubin), CK (creatine phosphokinase), $\mathrm{Na}$ (sodium), $\mathrm{K}$ (potassium), $\mathrm{Cl}$ (chloride), TG (triglyceride), and A/G (ratio of albumin to globulin) using a biochemical blood analyzer (Hitachi 7180, Hitachi, Japan). The blood was also analyzed for the WBC (white blood cell count), RBC (red blood cell count), Hb (hemoglobin concentration), HTC (hematocrit), MCV (mean corpuscular volume), $\mathrm{MCH}$ (mean corpuscular hemoglobin), MCHC (mean corpuscular hemoglobin concentration), RDW (red cell distribution width), PLT (platelet count), MPV (mean platelet volume), NE\# (number of neutrophils), NE\% (percent of neutrophils), LY\# (number of lymphocytes), LY\% percent of lymphocytes), MO\# (number of monocytes), MO\% (percent of monocytes), EO\# (number of eosinophils), EO\% (percent of eosinophils), BA\# (number of basophils), and BA\% (percent of basophils) using a blood cell counter (Hemavet 0950, CDC Tech., USA).

\section{Erythrocyte aggregation test}

To evaluate aggregation of red blood cells or blood coagulation attributable to the gold nanoparticles, 3.2\% sodium citrate was used for anticoagulation, and the activated partial thromboplastin time (APPT) and prothrombin time (PT) measured using a blood coagulation analyzer (ACL 7000, Instrumentation Laborato Co., U.S.A.).

\section{Kidney function test}

Since a gender difference in silver accumulation was previously noted in kidneys [14,15], kidney function was measured based on the $\mathrm{N}$-acetyl-beta- $\mathrm{D}$-glucosaminidase (NAG) and protein in the urine using metabolic cages for 5 rats from each exposure group and the control.

\section{Organ Weights and Histopathology}

After collecting blood samples, rats were killed by cervical dislocation, and all the organs carefully removed, including the adrenal glands, bladder, testes, ovaries, uterus, epididymis, seminal vesicle, heart, thymus, thyroid gland, trachea, esophagus, tongue, prostate, lungs, nasal cavity, kidneys, spleen, liver, pancreas, brain and olfactory bulb. The organs were then weighed, fixed in a $10 \%$ formalin solution containing neutral phosphate-buffered saline, embedded in paraffin, stained with hematoxylin and eosin, and examined under light microscopy.

\section{Lung Function Testing}

The lung function of four rats from each dose group was evaluated every week during the 90-day exposure using a ventilated bias flow whole-body plethysmograph (WBP) (SFT3816, Buxco Electronics, Sharon, CT) that consisted of a reference chamber and animal chamber interconnected by a pressure transducer (MAX1320, 
Buxco Electronics, Sharon, CT; [15]). Measurements taken during pulmonary function testing included the tidal volume (TV, $\mathrm{ml})$, minute volume $(\mathrm{MV}, \mathrm{ml} / \mathrm{min})$, respiratory frequency (BPM, breath/min), inspiration time $(\mathrm{Ti}, \mathrm{s})$, expiration time (Te, s), peak inspiration flow (PIF, $\mathrm{ml} / \mathrm{s}$ ), and peak expiration flow (PEF, $\mathrm{ml} / \mathrm{s}$ ). After the last 6-hour exposure, the rats were placed in an animal chamber, left for $40 \mathrm{~min}$ to stabilize, and measurements were taken in the whole-body plethysmograph for 5 minutes.

\section{Bronchoalveolar Lavage (BAL) Cell Evaluation}

The same animals that were used for lung function testing were also subjected to a BAL at the end of the 90-day exposure period. The rats were deeply anesthetized with an overdose of sodium pentobarbital. Blood was collected from the abdominal aorta and the rats were then exsanguinated by severing the abdominal aorta. The lungs were lavaged 14 times with $3 \mathrm{ml}$ aliquots of a warm calcium- and magnesium-free phosphate buffer solution (PBS), $\mathrm{pH}$ 7.4. The samples were also centrifuged for $7 \mathrm{~min}$ at $500 \times \mathrm{g}$ and the cell-free BAL fluid discarded. The cell pellets from all washes for each rat were then combined, washed, and resuspended in $1 \mathrm{ml}$ of a phosphate buffered saline (PBS) buffer and evaluated [16-20]. Total cell numbers were determined using a hemacytometer. The cells were first smeared and then stained with the Wright Giemsa Sure Stain [21] to allow a count of the total number of cells, macrophages, polymorphonuclear cells (PMNs), and lymphocytes. BAL levels of total protein, albumin, and LDH were also measured using a blood biochemical analyzer (Hitachi 7180, Hitachi, Japan).

\section{Determination of Tissue Gold}

After necropsy, samples of the blood $(0.5 \mathrm{ml})$, liver, lungs, brain, kidneys, and olfactory bulb were analyzed to determine organ distribution of gold. Care was taken to avoid contaminating tissues with gold from the fur and skin of each animal. After wet digestion using a flameless method, the tissue concentrations of gold were analyzed using an atomic absorption spectrophotometer equipped with a Zeeman graphite furnace (Perkin Elmer 5100ZL, Zeeman furnace module, USA) based on the NIOSH 7300 method [22]. The detection limit was 2 $\mathrm{ppb}$ and the limit of quantification was $7 \mathrm{ppb}$.

\section{Statistical analysis}

All results are expressed as means \pm standard error (SE). An analysis of variance (ANOVA) test and Duncan's multiple range tests were used to compare the body weights, bronchoalveolar lavage cell distributions, lung function test parameters, and all other comparisons for the three dose groups with those for the control rats.
Histopatholgic results were analyzed using a Chi-square analysis. The level of significance was set at $\mathrm{p}<0.05$ and $\mathrm{p}<0.01$.

\section{Results}

\section{Gold nanoparticle distribution}

Nanoparticle distributions in chambers are shown in Table 1. For the high-concentration chamber, the geometric mean diameter (GMD), concentration, and surface area of the gold nanoparticles measured by the DMAS were $5.06 \mathrm{~nm}, 1.85 \times 10^{6}$ particles $/ \mathrm{cm}^{3}, 20.02$ $\mu \mathrm{g} / \mathrm{m}^{3}$, and $3.64 \times 10^{8} \mathrm{~nm}^{2} / \mathrm{cm}^{3}$, respectively; for the middle-concentration chamber were $4.12 \mathrm{~nm}, 2.36 \times 10^{5}$ particles $/ \mathrm{cm}^{3}, 0.38 \mu \mathrm{g} / \mathrm{m}^{3}$ and $1.68 \times 10^{7} \mathrm{~nm}^{2} / \mathrm{cm}^{3}$, respectively, and for the low-concentration chamber were $4.3 \mathrm{~nm}, 2.36 \times 10^{4}$ particles $/ \mathrm{cm}^{3}, 0.04 \mu \mathrm{g} / \mathrm{m}^{3}$, and $1.9 \times 10^{6} \mathrm{~nm}^{2} / \mathrm{cm}^{3}$, respectively. The gold nanoparticles observed by TEM were spheroid in shape in both nonaggregated and non-agglomerated forms, with diameters under $6 \mathrm{~nm}$ (Figure 1,A-C). TEM-EDX analysis indicated that only elemental gold was present (Figure 1D). The diameters were log normally distributed between 1 and 6 $\mathrm{nm}$, and the CMD (count median diameter) and GSD were $2.47 \mathrm{~nm}$ and 1.42, respectively (Figure 2). The distribution of gold nanoparticles was well maintained during the 90-day exposure period, as shown in Figure 3.

\section{Animal Observation, Food Consumption, and Effect on Body and Organ Weights}

No significant gross effects were observed during the 90-day exposure period. There was a loss of hair on the front left leg of one male in the high dose group at 9 weeks and a loss of hair on both front legs of one female in the high dose group at 12 weeks of exposure. There was no apparent reason for the loss of hair in these two animals. In our experience, hair loss in isolated animals is not an unusual event. Examination of the eyes of control and high-dose animals did not reveal any effects. Food intake increased in the high-dose male group when compared to the control $(\mathrm{p}<0.05)$, middledose $(\mathrm{p}<0.05)$, and low-dose $(\mathrm{p}<0.01)$ groups (Figure $4 \mathrm{~A})$. Food intake also increased in the low-dose female group when compared to the control $(\mathrm{p}<0.01)$, middledose $(\mathrm{p}<0.01)$, and high-dose $(\mathrm{p}<0.05)$ groups (Figure 4B). While there were no significant changes in the body weights of the male rats (Figure 5A), there was a significant body weight gain in the low-dose female group ( $\mathrm{p}<0.05$ ) when compared to the control, middledose, and high-dose groups after 4 weeks of exposure (Figure 5B). There was a $0.3 \times 0.2 \mathrm{~cm}$ white mass in the right kidney of one rat from the high-dose group. No significant organ weight changes were observed in either the male or female rats at the conclusion of the study (Table 2 and 3). 
Table 1 Distribution of gold nanoparticles (mean \pm S.E.).

\begin{tabular}{|c|c|c|c|c|c|}
\hline Group & Site & $\operatorname{Diameter}^{\dagger}(\mathrm{nm})$ & Number (particles $/ \mathrm{cm}^{3}$ ) & Surface $\left(\mathrm{nm}^{2} / \mathrm{cm}^{3}\right)$ & Mass $\left(\mu \mathrm{g} / \mathrm{m}^{3}\right)$ \\
\hline Control & & 0 & 0 & 0 & 0 \\
\hline \multirow{3}{*}{ Low } & $U p^{a}$ & $4.29(1.69)$ & $2.46 \times 10^{4} \pm 2.38 \times 10^{2}$ & $1.96 \times 10^{6} \pm 3.28 \times 10^{4}$ & $0.04 \pm 0.00$ \\
\hline & Down $^{b}$ & $4.32(1.64)$ & $2.21 \times 10^{4} \pm 2.98 \times 10^{2}$ & $1.81 \times 10^{6} \pm 4.03 \times 10^{4}$ & $0.04 \pm 0.00$ \\
\hline & Total & $4.30(1.67)$ & $2.36 \times 10^{4} \pm 1.90 \times 10^{2}$ & $1.90 \times 10^{6} \pm 2.55 \times 10^{4}$ & $0.04 \pm 0.00$ \\
\hline \multirow{3}{*}{ Middle } & Up & $4.11(1.35)$ & $2.40 \times 10^{5} \pm 1.98 \times 10^{3}$ & $1.71 \times 10^{7} \pm 2.24 \times 10^{5}$ & $0.38 \pm 0.02$ \\
\hline & Down & $4.13(1.41)$ & $2.29 \times 10^{5} \pm 2.59 \times 10^{3}$ & $1.53 \times 10^{7} \pm 2.05 \times 10^{5}$ & $0.38 \pm 0.03$ \\
\hline & Total & $4.12(1.38)$ & $2.36 \times 10^{5} \pm 2.32 \times 10^{3}$ & $1.68 \times 10^{7} \pm 1.66 \times 10^{5}$ & $0.38 \pm 0.02$ \\
\hline \multirow{3}{*}{ High } & Up & $4.96(1.77)$ & $1.98 \times 10^{6} \pm 1.85 \times 10^{4}$ & $3.67 \times 10^{8} \pm 6.35 \times 10^{7}$ & $20.14 \pm 0.64$ \\
\hline & Down & $5.20(1.93)$ & $1.66 \times 10^{6} \pm 1.53 \times 10^{4}$ & $3.60 \times 10^{8} \pm 7.51 \times 10^{6}$ & $20.80 \pm 0.73$ \\
\hline & Total & $5.06(1.86)$ & $1.85 \times 10^{6} \pm 1.36 \times 10^{4}$ & $3.64 \times 10^{8} \pm 4.85 \times 10^{6}$ & $20.02 \pm 0.41$ \\
\hline
\end{tabular}

†: GM(GSD)

a: breathing zone,

b: below breathing zone.

Control chamber indicates number of particles passed after HEPA filter
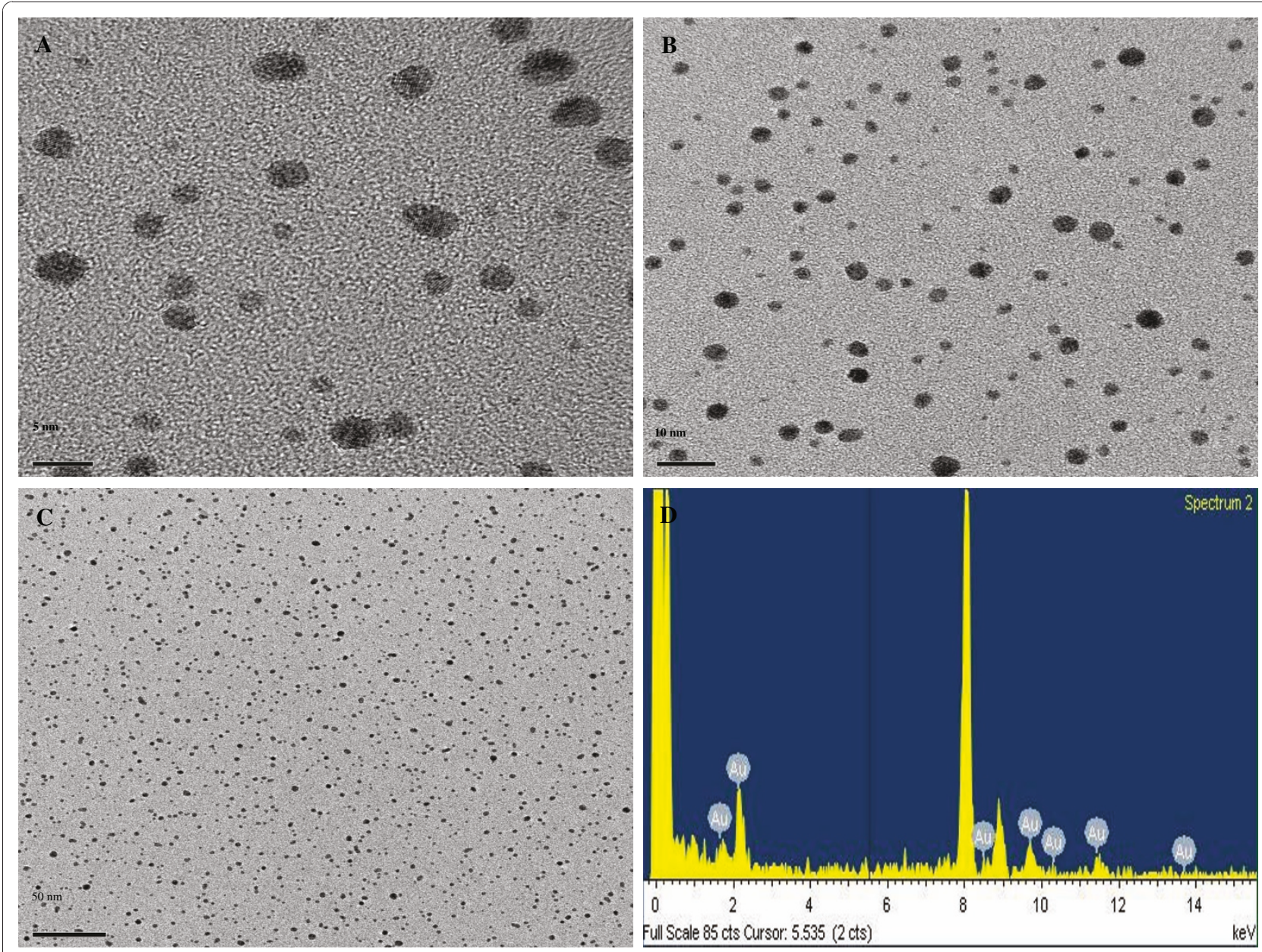

Figure 1 TEM morphology of gold nanoparticles and EDX spectrometer pattern. (A-C) Field Emission-Transmission Electron Microscope $(\times$ 100,000), (D) EDX spectrometer 

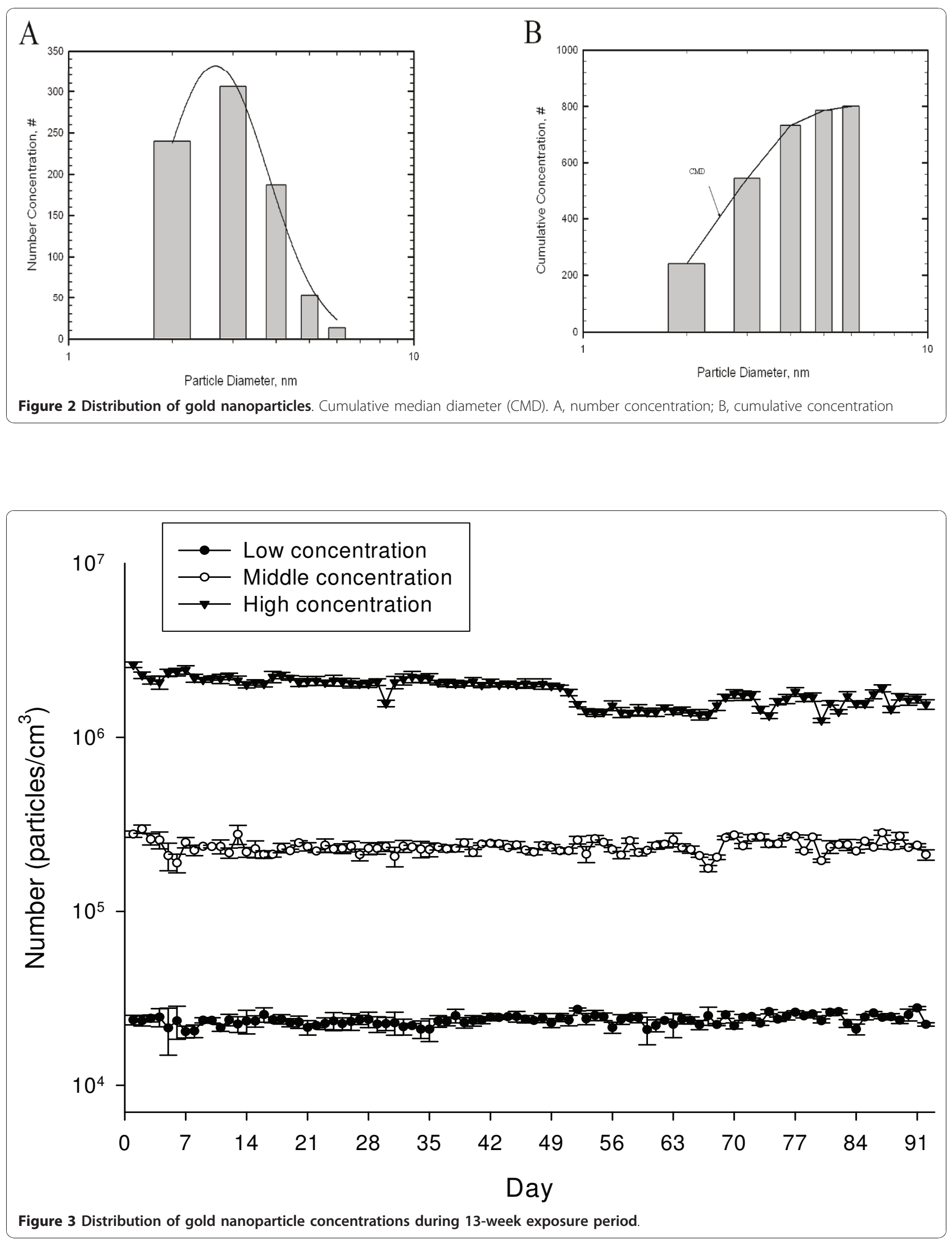


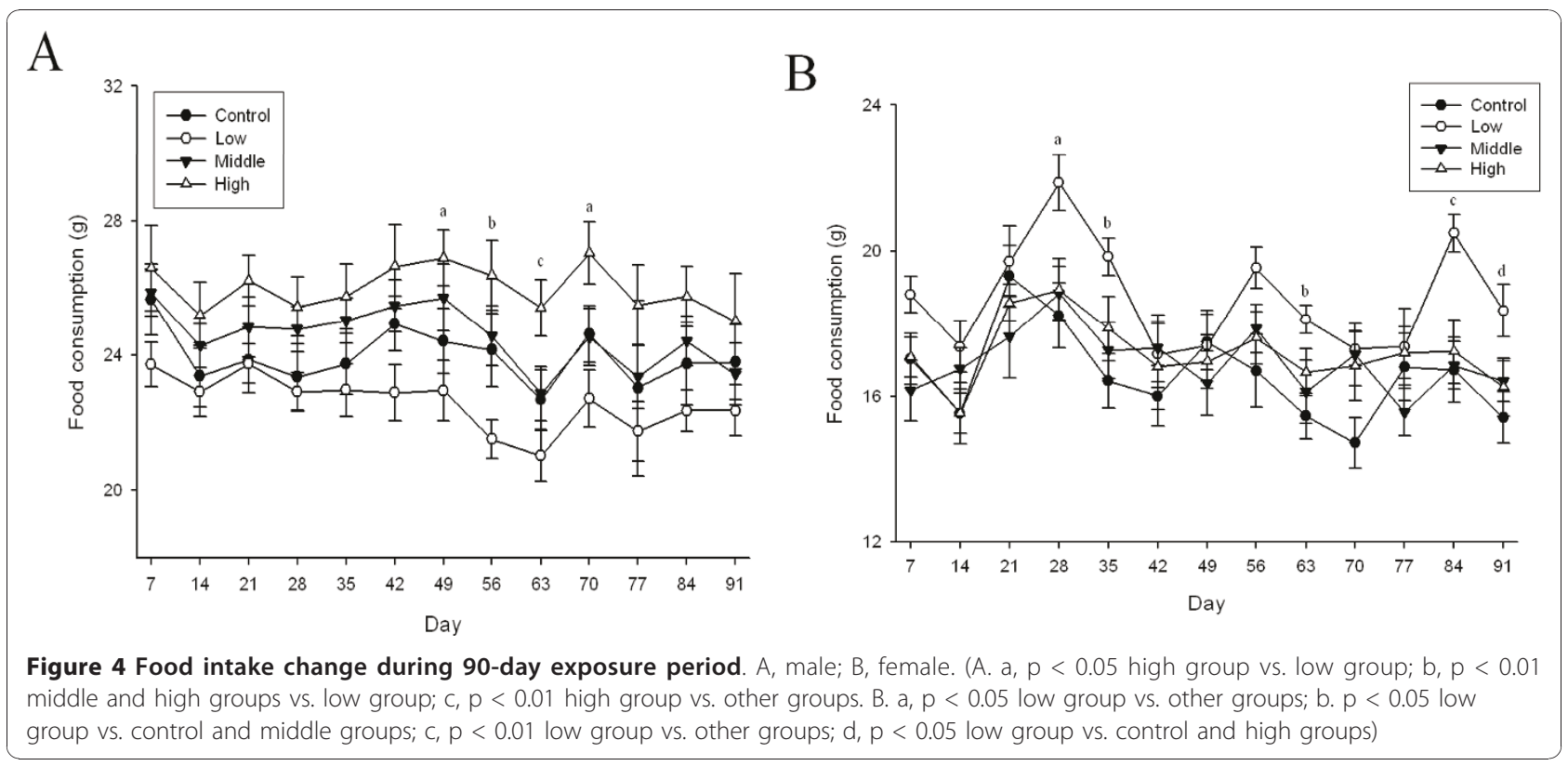

Effects on Hematology and Blood Biochemistry

No significant dose-related differences were observed for the hematology values (Table 4 and 5) and blood biochemical measurements (Table 6 and 7).

\section{Gold Distribution in Tissue}

Increases in gold concentrations in the lung tissue from the high dose group as compared to controls were statistically significant $(p<0.01)$ and increased with dose in both the males and females (Table 8 and 9). Gold concentrations in kidneys also increased in a dose dependent manner with statistical significance $(\mathrm{p}<$ $0.01)$. Interestingly, there was gender-related difference in gold concentrations in the kidneys (Table 8 and 9), with female kidneys showing more gold accumulation than the male kidneys. Apart from the lungs and kidneys, no dose-dependent increase of gold nanoparticles was found in the blood, liver, or olfactory bulb from both the male and female rats. Gold levels in blood were less than the tissues measured so that any small amount of contamination of other tissues with blood or blood contained in tissue was unlikely to greatly

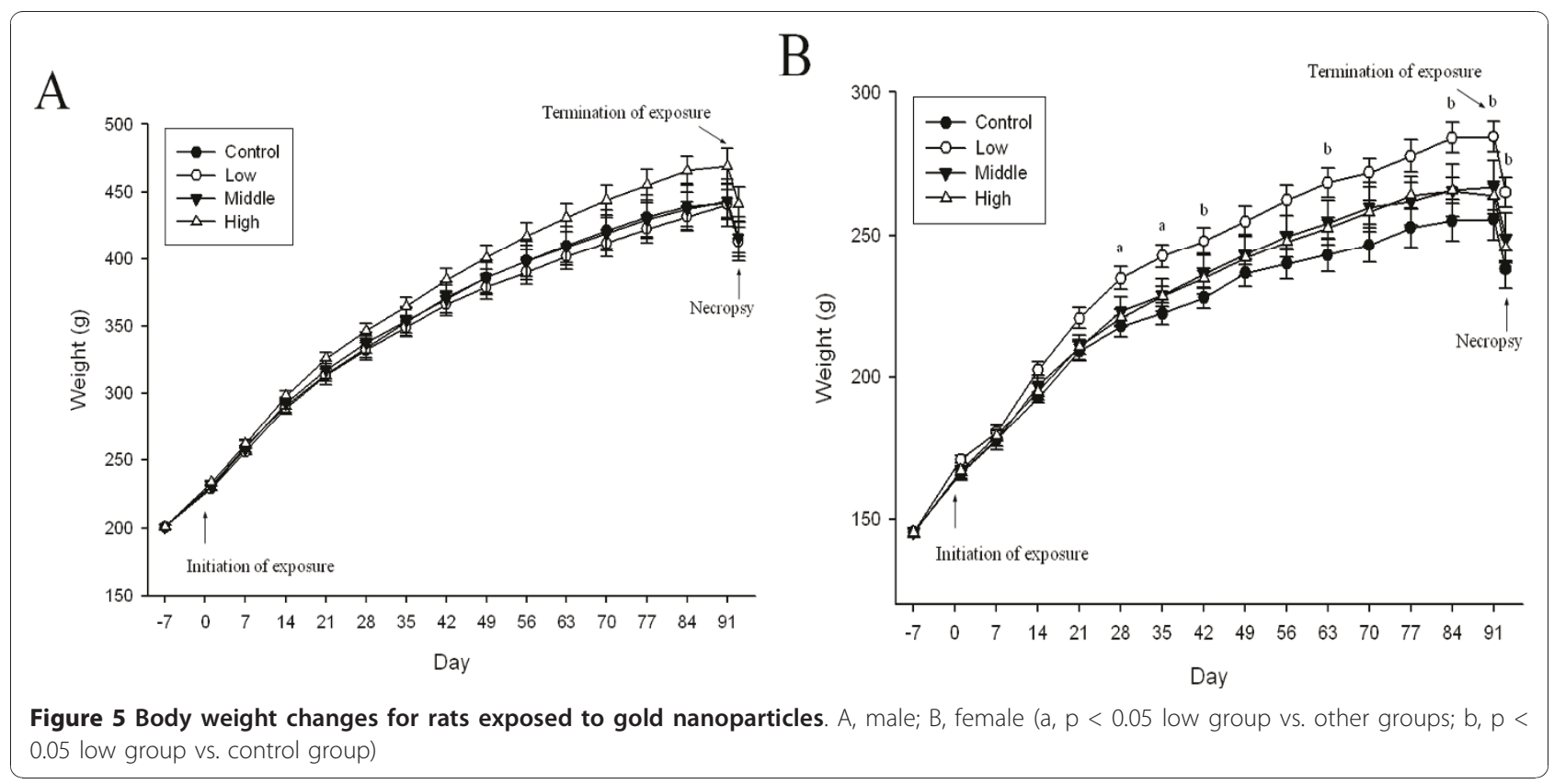


Table 2 Relative organ weights of male rats (\% body weight)

\begin{tabular}{lcccc}
\hline GROUP: (mean \pm S.E) & Control & Low & Middle & High \\
\hline Body weight & $415.08 \pm 7.79(10)$ & $412.81 \pm 10.83(10)$ & $415.93 \pm 11.72(10)$ & $440.81 \pm 12.48(10)$ \\
Testis (Left) & $0.41 \pm 0.01(8)$ & $0.44 \pm 0.02(10)$ & $0.45 \pm 0.01(10)$ & $0.41 \pm 0.02(8)$ \\
Testis (Right) & $0.40 \pm 0.01(8)$ & $0.43 \pm 0.02(10)$ & $0.45 \pm 0.01(10)$ & $0.40 \pm 0.02(8)$ \\
Kidney (Left) & $0.27 \pm 0.01(8)$ & $0.28 \pm 0.00(10)$ & $0.29 \pm 0.01(10)$ & $0.26 \pm 0.01(8)$ \\
Kidney (Right) & $0.28 \pm 0.01(8)$ & $0.29 \pm 0.01(10)$ & $0.29 \pm 0.01(10)$ & $0.28 \pm 0.01(8)$ \\
Spleen & $0.17 \pm 0.01(8)$ & $0.17 \pm 0.01(10)$ & $0.17 \pm 0.01(10)$ & $0.17 \pm 0.01(8)$ \\
Liver & $2.60 \pm 0.05(8)$ & $2.52 \pm 0.05(10)$ & $2.61 \pm 0.05(10)$ & $2.56 \pm 0.05(8)$ \\
Adrenal gland (Left) & $0.007 \pm 0.000(8)$ & $0.007 \pm 0.001(10)$ & $0.007 \pm 0.000(10)$ & $0.007 \pm 0.000(8)$ \\
Adrenal gland (Right) & $0.007 \pm 0.000(8)$ & $0.007 \pm 0.000(10)$ & $0.007 \pm 0.001(10)$ & $0.007 \pm 0.001(8)$ \\
Heart & $0.27 \pm 0.01(8)$ & $0.28 \pm 0.00(10)$ & $0.28 \pm 0.01(10)$ & $0.27 \pm 0.00(8)$ \\
Thymus & $0.08 \pm 0.01(8)$ & $0.08 \pm 0.00(10)$ & $0.09 \pm 0.01(10)$ & $0.08 \pm 0.01(8)$ \\
Lung (Left) & $0.12 \pm 0.00(6)$ & $0.12 \pm 0.00(6)$ & $0.11 \pm 0.00(6)$ & $0.11 \pm 0.00(6)$ \\
Kung (Right) & $0.22 \pm 0.00(6)$ & $0.23 \pm 0.00(6)$ & $0.21 \pm 0.00(6)$ & $0.21 \pm 0.00(6)$ \\
Brain & $0.49 \pm 0.01(8)$ & $0.51 \pm 0.01(10)$ & $0.51 \pm 0.01(10)$ & $0.47 \pm 0.01(8)$ \\
Olfactory bulb & $0.017 \pm 0.002(8)$ & $0.027 \pm 0.002(10)$ & $0.023 \pm 0.003(10)$ & $0.022 \pm 0.001(8)$ \\
Hypophysis & $0.002 \pm 0.000(8)$ & $0.002 \pm 0.000(10)$ & $0.002 \pm 0.000(10)$ & $0.002 \pm 0.000(8)$ \\
\hline
\end{tabular}

( ): number of animals

influence tissue measurements of gold content. There was a significant increase in gold nanoparticle concentration only in brain tissue from high-dose females (Table 9).

\section{Pulmonary Inflammation}

Total number of cells, alveolar macrophages, polymorphonuclear cells (PMN), and lymphocytes in BAL fluid after 90-days of exposure are shown in Figure 6. When compared to the control group, there were no significant changes in total cell numbers, alveolar macrophages, PMN, or lymphocytes of any exposed males. There was a significant increase in lymphocytes in middle and high-dose females (Figure 6B). Albumin, $\mathrm{LDH}$, and total protein in BAL did not increase significantly in either male or female rats (data not shown).

\section{Pulmonary Function Testing}

Among the pulmonary function test parameters, there were significant changes in tidal volume and minute volume during the 90-days of gold nanoparticle exposure $(p<0.01-0.05)$ (Figures 7,A and 7B and 8,A and $8 \mathrm{~B})$. Dose-dependent tidal volume decreases in male rats led to minute volume decreases in the high-dose animals. A tendency towards a dose-dependent decrease in

Table 3 Relative organ weights of female rats (\% body weight)

\begin{tabular}{lcccc}
\hline GROUP: (mean \pm S.E) & Control & Low & Middle & High \\
\hline Body weight & $237.53 \pm 6.76(10)$ & $264.88 \pm 5.19(10)$ & $248.82 \pm 9.05(10)$ & $245.26 \pm 4.91(10)$ \\
Ovary (Left) & $0.020 \pm 0.002(10)$ & $0.021 \pm 0.001(10)$ & $0.019 \pm 0.001(10)$ & $0.020 \pm 0.001(10)$ \\
Ovary (Right) & $0.021 \pm 0.001(10)$ & $0.021 \pm 0.001(10)$ & $0.021 \pm 0.001(10)$ & $0.021 \pm 0.002(10)$ \\
Kidney (Left) & $0.29 \pm 0.01(10)$ & $0.28 \pm 0.00(10)$ & $0.30 \pm 0.01(10)$ & $0.29 \pm 0.01(10)$ \\
Kidney (Right) & $0.30 \pm 0.01(10)$ & $0.29 \pm 0.01(10)$ & $0.30 \pm 0.01(10)$ & $0.29 \pm 0.01(10)$ \\
Spleen & $0.20 \pm 0.01(10)$ & $0.18 \pm 0.01(10)$ & $0.19 \pm 0.01(10)$ & $0.18 \pm 0.01(10)$ \\
Liver & $2.42 \pm 0.03(10)$ & $2.43 \pm 0.04(10)$ & $2.54 \pm 0.06(10)$ & $2.44 \pm 0.05(10)$ \\
Adrenal gland (Left) & $0.013 \pm 0.001(10)$ & $0.013 \pm 0.001(10)$ & $0.012 \pm 0.001(10)$ & $0.013 \pm 0.001(10)$ \\
Adrenal gland (Right) & $0.013 \pm 0.001(10)$ & $0.013 \pm 0.001(10)$ & $0.012 \pm 0.001(10)$ & $0.015 \pm 0.002(10)$ \\
Heart & $0.34 \pm 0.01(10)$ & $0.32 \pm 0.01(10)$ & $0.32 \pm 0.01(10)$ & $0.33 \pm 0.01(10)$ \\
Thymus & $0.12 \pm 0.00(10)$ & $0.14 \pm 0.01(10)$ & $0.12 \pm 0.01(10)$ & $0.13 \pm 0.01(10)$ \\
Lung (Left) & $0.18 \pm 0.03(6)$ & $0.17 \pm 0.03(6)$ & $0.17 \pm 0.02(6)$ & $0.16 \pm 0.00(6)$ \\
Kung (Right) & $0.36 \pm 0.05(6)$ & $0.31 \pm 0.03(6)$ & $0.32 \pm 0.04(6)$ & $0.30 \pm 0.01(6)$ \\
Brain & $0.84 \pm 0.02(10)$ & $0.76 \pm 0.02(10)$ & $0.80 \pm 0.03(10)$ & $0.82 \pm 0.02(10)$ \\
Olfactory bulb & $0.040 \pm 0.003(10)$ & $0.034 \pm 0.002(10)$ & $0.041 \pm 0.002(10)$ & $0.039 \pm 0.002(10)$ \\
Hypophysis & $0.005 \pm 0.001(10)$ & $0.005 \pm 0.000(10)$ & $0.006 \pm 0.001(10)$ & $0.006 \pm 0.001(10)$ \\
\hline
\end{tabular}

( ): number of animals 
Table 4 Hematological values for male rats

\begin{tabular}{lcccc}
\hline GROUP: $($ mean \pm S.E) & Control & Low & Middle & High \\
\hline $\mathrm{WBC}^{1}(\mathrm{~K} / \mu \mathrm{L})$ & $5.17 \pm 0.44(8)$ & $6.42 \pm 0.64(10)$ & $4.80 \pm 0.27(10)$ & $4.67 \pm 0.20(8)$ \\
$\mathrm{NE}^{2}(\mathrm{~K} / \mu \mathrm{L})$ & $1.56 \pm 0.14(8)$ & $1.67 \pm 0.20(10)$ & $1.41 \pm 0.11(10)$ & $1.41 \pm 0.10(8)$ \\
$\mathrm{LY}^{3}(\mathrm{~K} / \mu \mathrm{L})$ & $3.36 \pm 0.36(8)$ & $4.41 \pm 0.42(10)$ & $3.11 \pm 0.22(10)$ & $3.06 \pm 0.18(8)$ \\
$\mathrm{MO}^{4}(\mathrm{~K} / \mu \mathrm{L})$ & $0.24 \pm 0.03(8)$ & $0.32 \pm 0.04(10)$ & $0.27 \pm 0.17(10)$ & $0.19 \pm 0.02(8)$ \\
$\mathrm{EO}^{5}(\mathrm{~K} / \mu \mathrm{L})$ & $0.005 \pm 0.002(8)$ & $0.018 \pm 0.013(10)$ & $0.006 \pm 0.002(10)$ & $0.014 \pm 0.003(8)$ \\
$\mathrm{BA}^{6}(\mathrm{~K} / \mu \mathrm{L})$ & $0.000 \pm 0.000(8)$ & $0.008 \pm 0.008(10)$ & $0.000 \pm 0.000(10)$ & $0.003 \pm 0.002(8)$ \\
$\mathrm{NE}^{7}(\%)$ & $30.72 \pm 2.39(8)$ & $25.71 \pm 0.98(10)$ & $29.553 \pm 1.70(10)$ & $30.28 \pm 2.07(8)$ \\
$\mathrm{LY}^{8}(\%)$ & $64.45 \pm 2.38(8)$ & $68.97 \pm 1.18(10)$ & $64.52 \pm 1.77(10)$ & $65.39 \pm 1.96(8)$ \\
$\mathrm{MO}^{9}(\%)$ & $4.71 \pm 0.50(8)$ & $5.05 \pm 0.48(10)$ & $5.78 \pm 0.42(10)$ & $4.00 \pm 0.27(8)$ \\
$\mathrm{EO}^{10}(\%)$ & $0.10 \pm 0.03(8)$ & $0.20 \pm 0.11(10)$ & $0.15 \pm 0.05(10)$ & $0.30 \pm 0.05(8)$ \\
$\mathrm{BA}^{11}(\%)$ & $0.15 \pm 0.01(8)$ & $0.08 \pm 0.07(10)$ & $0.01 \pm 0.00(10)$ & $0.03 \pm 0.02(8)$ \\
$\mathrm{RBC}^{12}(\mathrm{M} / \mu \mathrm{L})$ & $8.87 \pm 0.11(8)$ & $8.67 \pm 0.33(10)$ & $9.18 \pm 0.16(10)$ & $9.09 \pm 0.17(8)$ \\
$\mathrm{Hb}^{13}(\mathrm{~g} / \mathrm{dL})$ & $17.68 \pm 0.23(8)$ & $17.33 \pm 0.18(10)$ & $18.26 \pm 0.24(10)$ & $17.91 \pm 0.22(8)$ \\
$\mathrm{HCT}^{14}(\%)$ & $36.71 \pm 1.07(8)$ & $36.68 \pm 0.95(10)$ & $38.26 \pm 0.98(10)$ & $37.66 \pm 1.18(8)$ \\
$\mathrm{MCV}^{15}(\mathrm{fL})$ & $41.35 \pm 0.97(8)$ & $42.30 \pm 0.86(10)$ & $41.65 \pm 0.78(10)$ & $41.46 \pm 1.13(8)$ \\
$\mathrm{MCH}^{16}(\mathrm{pg})$ & $19.95 \pm 0.34(8)$ & $20.00 \pm 0.25(10)$ & $19.92 \pm 0.36(10)$ & $19.73 \pm 0.28(8)$ \\
$\mathrm{MCHC}^{17}(\mathrm{~g} / \mathrm{dL})$ & $48.39 \pm 1.29(8)$ & $47.53 \pm 1.31(10)$ & $47.98 \pm 1.32(10)$ & $47.86 \pm 1.44(8)$ \\
$\mathrm{RDW}^{18}(\%)$ & $20.74 \pm 0.94(8)$ & $21.24 \pm 1.08(10)$ & $21.76 \pm 1.09(10)$ & $20.84 \pm 0.97(8)$ \\
$\mathrm{PLT}^{19}(\mathrm{~K} / \mu \mathrm{L})$ & $949.88 \pm 50.68(8)$ & $862.70 \pm 41.33(10)$ & $894.30 \pm 32.72(10)$ & $882.38 \pm 42.43(8)$ \\
$\mathrm{MPV}^{20}(\mathrm{fL})$ & $6.43 \pm 0.09(8)$ & $6.67 \pm 0.12(10)$ & $6.61 \pm 0.11(10)$ & $6.44 \pm 0.16(8)$ \\
\hline
\end{tabular}

( ): number of animals

1, White blood cells; 2, Neutrophils; 3, Lymphocytes; 4, Monocytes; 5, Eosinophils; 6, Basophils; 7, Percent of neutrophils; 8, Percent of lymphocytes; 9, Percent of monocytes; 10, Percent of eosinophils; 11, Percent of basophils; 12, Red blood cells; 13, Hemoglobin; 14, Hematocrits; 15, Mean corpuscular volume; 16, Mean corpuscular hemoglobin; 17, Mean corpuscular hemoglobin concentration; 18, Red cell distribution width; 19, Platelet; 20, Mean platelet volume

Table 5 Hematological values for female rats

\begin{tabular}{lcccc}
\hline GROUP: (mean \pm S.E) & Control & Low & Middle & High \\
\hline $\mathrm{WBC}^{1}(\mathrm{~K} / \mu \mathrm{L})$ & $3.60 \pm 0.43(10)$ & $2.99 \pm 0.30(10)$ & $3.25 \pm 0.36(10)$ & $3.39 \pm 0.39(10)$ \\
$\mathrm{NE}^{2}(\mathrm{~K} / \mu \mathrm{L})$ & $0.96 \pm 0.11(10)$ & $0.81 \pm 0.08(10)$ & $0.79 \pm 0.08(10)$ & $1.02 \pm 0.15(10)$ \\
$\mathrm{LY}^{3}(\mathrm{~K} / \mu \mathrm{L})$ & $2.50 \pm 0.33(10)$ & $2.06 \pm 0.22(10)$ & $2.31 \pm 0.28(10)$ & $2.24 \pm 0.25(10)$ \\
$\mathrm{MO}^{4}(\mathrm{~K} / \mu \mathrm{L})$ & $0.13 \pm 0.03(10)$ & $0.12 \pm 0.02(10)$ & $0.14 \pm 0.02(10)$ & $0.12 \pm 0.02(10)$ \\
$E O^{5}(\mathrm{~K} / \mu \mathrm{L})$ & $0.007 \pm 0.004(10)$ & $0.004 \pm 0.002(10)$ & $0.006 \pm 0.002(10)$ & $0.006 \pm 0.002(10)$ \\
$\mathrm{BA}^{6}(\mathrm{~K} / \mu \mathrm{L})$ & $0.002 \pm 0.002(10)$ & $0.000 \pm 0.000(10)$ & $0.001 \pm 0.001(10)$ & $0.000 \pm 0.000(10)$ \\
$\mathrm{NE}^{7}(\%)$ & $27.22 \pm 2.34(10)$ & $27.42 \pm 1.37(10)$ & $24.97 \pm 1.43(10)$ & $29.81 \pm 1.87(10)$ \\
$\mathrm{LY}^{8}(\%)$ & $26.99 \pm 2.13(10)$ & $68.23 \pm 1.57(10)$ & $70.62 \pm 1.40(10)$ & $66.50 \pm 1.68(10)$ \\
$\mathrm{MO}^{9}(\%)$ & $3.47 \pm 0.43(10)$ & $4.16 \pm 0.51(10)$ & $4.17 \pm 0.31(10)$ & $3.49 \pm 0.53(10)$ \\
$E O^{10}(\%)$ & $0.24 \pm 0.09(10)$ & $0.17 \pm 0.04(10)$ & $0.20 \pm 0.05(10)$ & $0.18 \pm 0.04(10)$ \\
$\mathrm{BA}^{11}(\%)$ & $0.08 \pm 0.04(10)$ & $0.02 \pm 0.01(10)$ & $0.04 \pm 0.02(10)$ & $0.03 \pm 0.01(10)$ \\
$\mathrm{RBC}^{12}(\mathrm{M} / \mu \mathrm{L})$ & $8.19 \pm 0.09(10)$ & $7.94 \pm 0.15(10)$ & $8.07 \pm 0.05(10)$ & $7.96 \pm 0.11(10)$ \\
$\mathrm{Hb}^{13}(\mathrm{~g} / \mathrm{dL})$ & $16.99 \pm 0.22(10)$ & $16.70 \pm 0.28(10)$ & $16.74 \pm 0.12(10)$ & $16.61 \pm 0.27(10)$ \\
$\mathrm{HCT}^{14}(\%)$ & $39.93 \pm 0.67(10)$ & $39.41 \pm 0.71(10)$ & $39.54 \pm 0.41(10)$ & $39.24 \pm 0.71(10)$ \\
$\mathrm{MCV}^{15}(\mathrm{fL})$ & $48.82 \pm 0.86(10)$ & $49.70 \pm 0.39(10)$ & $48.99 \pm 0.65(10)$ & $49.30 \pm 0.50(10)$ \\
$\mathrm{MCH}^{16}(\mathrm{pg})$ & $20.77 \pm 0.29(10)$ & $21.06 \pm 0.22(10)$ & $20.74 \pm 0.18(10)$ & $20.87 \pm 0.19(10)$ \\
$\mathrm{MCHC}^{17}(\mathrm{~g} / \mathrm{dL})$ & $42.58 \pm 0.37(10)$ & $42.38 \pm 0.31(10)$ & $42.37 \pm 0.36(10)$ & $42.35 \pm 0.26(10)$ \\
$\mathrm{RDW}^{18}(\%)$ & $22.75 \pm 1.22(10)$ & $22.58 \pm 1.27(10)$ & $22.52 \pm 1.10(10)$ & $23.94 \pm 1.34(10)$ \\
$\mathrm{PLT}^{19}(\mathrm{~K} / \mu \mathrm{L})$ & $693.10 \pm 68.03(10)$ & $762.70 \pm 25.24(10)$ & $729.00 \pm 15.91(10)$ & $764.90 \pm 14.86(10)$ \\
$\mathrm{MPV}^{20}(\mathrm{fL})$ & $6.45 \pm 0.15(10)$ & $6.66 \pm 0.17(10)$ & $6.47 \pm 0.14(10)$ & $6.36 \pm 0.20(10)$ \\
\hline
\end{tabular}

( ): number of animals

1, White blood cells; 2, Neutrophils; 3, Lymphocytes; 4, Monocytes; 5, Eosinophils; 6, Basophils; 7, Percent of neutrophils; 8, Percent of lymphocytes; 9, Percent of monocytes; 10, Percent of eosinophils; 11, Percent of basophils; 12, Red blood cells; 13, Hemoglobin; 14, Hematocrits; 15, Mean corpuscular volume; 16, Mean corpuscular hemoglobin; 17, Mean corpuscular hemoglobin concentration; 18, Red cell distribution width; 19, Platelet; 20, Mean platelet volume 
Table 6 Serum biochemical values for male rats

\begin{tabular}{|c|c|c|c|c|}
\hline GROUP: (mean \pm S.E) & Control & Low & Middle & High \\
\hline $\mathrm{ALB}^{1}(\mathrm{~g} / \mathrm{dL})$ & $2.43 \pm 0.02(8)$ & $2.42 \pm 0.02(10)$ & $2.50 \pm 0.03(10)$ & $2.56 \pm 0.05$ \\
\hline $\mathrm{ALP}^{2}(\mathrm{IU} / \mathrm{L})$ & $286.63 \pm 13.86(8)$ & $299.10 \pm 17.32(10)$ & $332.30 \pm 17.13(10)$ & $292.88 \pm 121.40$ \\
\hline $\mathrm{CA}^{3}(\mathrm{mg} / \mathrm{dL})$ & $9.18 \pm 0.12(8)$ & $9.09 \pm 0.04(10)$ & $15.02 \pm 0.75 b(10)$ & $18.93 \pm 0.14(8)$ \\
\hline $\mathrm{CHO}^{4}(\mathrm{mg} / \mathrm{dL})$ & $68.75 \pm 4.69(8)$ & $69.60 \pm 4.76(10)$ & $69.00 \pm 3.44(10)$ & $77.38 \pm 8.66(8)$ \\
\hline $\mathrm{CRE}^{5}(\mathrm{mg} / \mathrm{dL})$ & $0.53 \pm 0.05$ & $0.59 \pm 0.13(10)$ & $1.08 \pm 0.08 b(10)$ & $0.91 \pm 0.12(8)$ \\
\hline$\gamma-\mathrm{GT}^{6}(\mathrm{IU} / \mathrm{L})$ & $1.38 \pm 0.18(8)$ & $1.10 \pm 0.18(10)$ & $1.10 \pm 0.18(10)$ & $1.25 \pm 0.16(8)$ \\
\hline $\mathrm{GLU}^{7}(\mathrm{mg} / \mathrm{dL})$ & $158.13 \pm 9.34(8)$ & $175.40 \pm 9.56(10)$ & $188.50 \pm 13.55(10)$ & $162.38 \pm 7.81(8)$ \\
\hline $\mathrm{GOT}^{8}(\mathrm{IU} / \mathrm{L})$ & $124.25 \pm 16.27(8)$ & $105.10 \pm 8.97(10)$ & $108.60 \pm 9.89(10)$ & $102.13 \pm 14.7(8)$ \\
\hline $\mathrm{GPT}^{9}(\mathrm{IU} / \mathrm{L})$ & $66.63 \pm 14.49(8)$ & $49.30 \pm 2.50(10)$ & $54.90 \pm 4.18(10)$ & $51.50 \pm 6.76(8)$ \\
\hline $\mathrm{IP}^{10}(\mathrm{mg} / \mathrm{dL})$ & $6.54 \pm 0.31(8)$ & $6.73 \pm 0.24(10)$ & $6.60 \pm 0.20(10)$ & $6.20 \pm 0.23$ \\
\hline $\mathrm{LDH}^{11}(\mathrm{IU} / \mathrm{L})$ & $562.63 \pm 103.39(8)$ & $555.50 \pm 149.47(10)$ & $592.40 \pm 179.20(10)$ & $538.88 \pm 193.15$ \\
\hline$M G^{12}(\mathrm{mg} / \mathrm{dL})$ & $2.04 \pm 0.04$ & $2.12 \pm 0.05(10)$ & $2.05 \pm 0.05(10)$ & $2.03 \pm 0.05(8)$ \\
\hline $\mathrm{TP}^{13}(\mathrm{~g} / \mathrm{dL})$ & $6.00 \pm 0.07(8)$ & $5.92 \pm 0.02(10)$ & $6.11 \pm 0.05(10)$ & $6.25 \pm 0.09$ \\
\hline $\mathrm{UA}^{14}(\mathrm{mg} / \mathrm{dL})$ & $1.24 \pm 0.21$ & $1.39 \pm 0.10(10)$ & $1.62 \pm 0.26(10)$ & $1.04 \pm 0.10$ \\
\hline$B \cup N^{15}(\mathrm{mg} / \mathrm{dL})$ & $15.88 \pm 0.65(8)$ & $16.86 \pm 0.47(10)$ & $16.33 \pm 0.48(10)$ & $15.11 \pm 0.37(8)$ \\
\hline $\mathrm{TBIL}^{16}(\mathrm{mg} / \mathrm{dL})$ & $0.013 \pm 0.004$ & $0.008 \pm 0.005(10)$ & $0.001 \pm 0.008(10)$ & $0.010 \pm 0.008$ (8) \\
\hline $\mathrm{TG}^{17}(\mathrm{mg} / \mathrm{dL})$ & $37.25 \pm 6.87(8)$ & $40.60 \pm 9.24(10)$ & $38.80 \pm 6.00(10)$ & $41.75 \pm 5.54(8)$ \\
\hline $\mathrm{CK}^{18}(\mathrm{IU} / \mathrm{L})$ & $543.88 \pm 77.39(8)$ & $1001.20 \pm 213.75$ & $759.70 \pm 146.20(10)$ & $419.13 \pm 125.29(8)$ \\
\hline $\mathrm{Na}^{19}(\mathrm{mmol} / \mathrm{L})$ & $129.13 \pm 0.35(8)$ & $129.30 \pm 0.52(10)$ & $128.50 \pm 0.56(10)$ & $132.13 \pm 2.07(8)$ \\
\hline $\mathrm{K}^{20}(\mathrm{mmol} / \mathrm{L})$ & $2.80 \pm 0.07(8)$ & $3.02 \pm 0.10(10)$ & $3.21 \pm 0.15(10)$ & $2.80 \pm 0.10$ \\
\hline $\mathrm{Cl}^{21}(\mathrm{mmol} / \mathrm{L})$ & $97.25 \pm 0.31(8)$ & $97.70 \pm 0.21(10)$ & $97.10 \pm 0.35(10)$ & $99.75 \pm 1.49(8)$ \\
\hline
\end{tabular}

( ): number of animals

1, Albumin; 2, Alkaline phosphatase; 3, Calcium; 4, Cholesterol; 5, Creatinine; 6, Gamma glutamyl transpeptidase; 7, Glucose; 8, Glutamic oxalacetic transaminase; 9, Glutamic pyruvic transaminase; 10, Inorganic phosphorus; 11, Lactate Dehydrogenase; 12, Magnesium; 13, Total protein; 14, Uric acid; 15, Blood urea nitrogen; 16, Total bilirubin; 17, Triglyceride; 18, Creatine Kinase; 19, Sodium; 20, Potassium; 21, Chloride

Table 7 Serum biochemical values for female rats

\begin{tabular}{lcccc}
\hline GROUP: $(\mathrm{mean} \pm \mathrm{S} . \mathrm{E})$ & Control & Low & Middle & High \\
\hline $\mathrm{ALB}^{1}(\mathrm{~g} / \mathrm{dL})$ & $2.77 \pm 0.07(10)$ & $2.76 \pm 0.08(10)$ & $3.18 \pm 0.11(10)$ & $2.85 \pm 0.06(10)$ \\
$\mathrm{ALP}^{2}(\mathrm{IU} / \mathrm{L})$ & $223.20 \pm 16.90(10)$ & $200.80 \pm 22.94(10)$ & $167.70 \pm 9.59(10)$ & $227.20 \pm 13.71(10)$ \\
$\mathrm{CA}^{3}(\mathrm{mg} / \mathrm{dL})$ & $10.02 \pm 0.10(10)$ & $10.10 \pm 0.14(10)$ & $10.36 \pm 0.17(10)$ & $10.29 \pm 0.19(10)$ \\
$\mathrm{CHO}^{4}(\mathrm{mg} / \mathrm{dL})$ & $89.60 \pm 4.49(10)$ & $96.90 \pm 4.66(10)$ & $105.20 \pm 5.59(10)$ & $92.70 \pm 5.18(10)$ \\
$\mathrm{CRE}^{5}(\mathrm{mg} / \mathrm{dL})$ & $0.78 \pm 0.04(10)$ & $0.89 \pm 0.04(10)$ & $0.89 \pm 0.07(10)$ & $0.85 \pm 0.03(10)$ \\
$\gamma-\mathrm{GT}^{6}(\mathrm{IU} / \mathrm{L})$ & $0.10 \pm 0.10(10)$ & $0.20 \pm 0.13(10)$ & $0.00 \pm 0.00(10)$ & $0.40 \pm 0.22(10)$ \\
$\mathrm{GLU}^{7}(\mathrm{mg} / \mathrm{dL})$ & $134.70 \pm 5.24(10)$ & $143.30 \pm 4.82(10)$ & $154.50 \pm 8.04(10)$ & $135.90 \pm 5.23(10)$ \\
$\mathrm{GO}^{8}(\mathrm{IU} / \mathrm{L})$ & $69.00 \pm 2.21(10)$ & $141.20 \pm 48.60(10)$ & $77.40 \pm 4.96(10)$ & $82.30 \pm 5.17(10)$ \\
$\mathrm{GPT}^{9}(\mathrm{IU} / \mathrm{L})$ & $33.80 \pm 1.13(10)$ & $64.20 \pm 15.61(10)$ & $40.60 \pm 2.85(10)$ & $41.60 \pm 2.63(10)$ \\
$\mathrm{IP}^{10}(\mathrm{mg} / \mathrm{dL})$ & $5.66 \pm 0.23(10)$ & $5.64 \pm 0.49(10)$ & $5.58 \pm 0.40(10)$ & $5.55 \pm 0.29(10)$ \\
$\mathrm{LDH}^{11}(\mathrm{IU} / \mathrm{L})$ & $180.40 \pm 27.93(10)$ & $254.90 \pm 49.86(10)$ & $222.70 \pm 22.46(10)$ & $216.40 \pm 30.50(10)$ \\
$\mathrm{MG}^{12}(\mathrm{mg} / \mathrm{dL})$ & $2.06 \pm 0.04(10)$ & $2.04 \pm 0.04(10)$ & $2.13 \pm 0.07(10)$ & $2.03 \pm 0.04(10)$ \\
$\mathrm{TP}^{13}(\mathrm{~g} / \mathrm{dL})$ & $6.20 \pm 0.09(10)$ & $6.07 \pm 0.17(10)$ & $6.76 \pm 0.18(10)$ & $6.40 \pm 0.13(10)$ \\
$\mathrm{UA}^{14}(\mathrm{mg} / \mathrm{dL})$ & $1.07 \pm 0.11(10)$ & $1.22 \pm 0.09(10)$ & $1.14 \pm 0.06(10)$ & $0.99 \pm 0.08(10)$ \\
$\mathrm{BUN}^{15}(\mathrm{mg} / \mathrm{dL})$ & $17.86 \pm 0.74(10)$ & $18.08 \pm 0.61(10)$ & $18.99 \pm 0.90(10)$ & $17.52 \pm 0.87(10)$ \\
$\mathrm{TBIL}^{16}(\mathrm{mg} / \mathrm{dL})$ & $0.005 \pm 0.015(10)$ & $0.009 \pm 0.013(10)$ & $0.020 \pm 0.005(10)$ & $0.024 \pm 0.005(10)$ \\
$\mathrm{TG}^{17}(\mathrm{mg} / \mathrm{dL})$ & $8.50 \pm 1.28(10)$ & $9.40 \pm 0.93(10)$ & $11.20 \pm 1.83(10)$ & $6.90 \pm 0.57(10)$ \\
$\mathrm{CK}^{18}(\mathrm{IU} / \mathrm{L})$ & $269.20 \pm 64.71(10)$ & $265.50 \pm 24.99(10)$ & $341.30 \pm 68.11(10)$ & $212.30 \pm 26.38(10)$ \\
$\mathrm{Na}^{19}(\mathrm{mmol} / \mathrm{L})$ & $136.50 \pm 0.48(10)$ & $139.20 \pm 2.80(10)$ & $138.90 \pm 0.95(10)$ & $138.50 \pm 1.26(10)$ \\
$\mathrm{K}^{20}(\mathrm{mmol})$ & $3.69 \pm 0.12(10)$ & $3.56 \pm 0.10(10)$ & $3.62 \pm 0.07(10)$ & $3.48 \pm 0.09(10)$ \\
$\mathrm{Cl}^{21}(\mathrm{mmol} / \mathrm{L})$ & $102.00 \pm 0.33(10)$ & $103.40 \pm 2.05(10)$ & $103.00 \pm 0.86(10)$ & $102.80 \pm 1.17(10)$ \\
\hline
\end{tabular}

( ): number of animals

1, Albumin; 2, Alkaline phosphatase; 3, Calcium; 4, Cholesterol; 5, Creatinine; 6, Gamma glutamyl transpeptidase; 7, Glucose; 8, Glutamic oxalacetic transaminase; 9, Glutamic pyruvic transaminase; 10, Inorganic phosphorus; 11, Lactate Dehydrogenase; 12, Magnesium; 13, Total protein; 14, Uric acid; 15, Blood urea nitrogen; 16, Total bilirubin; 17, Triglyceride; 18, Creatine Kinase; 19, Sodium; 20, Potassium; 21, Chloride 
Table 8 Gold distribution in tissue of male rats after 90 -day gold nanoparticle exposure.

\begin{tabular}{lcccc}
\hline GROUP: (mean \pm S.E) & Control & Low & Middle & High \\
\hline Lungs (ng/g) & $14.33 \pm 1.25(5)$ & $17.41 \pm 1.49(5)$ & $36.88 \pm 1.69(5)$ & $2191.77 \pm 222.59^{\mathrm{a}}(5)$ \\
Liver (ng/g) & $13.58 \pm 0.69(5)$ & $13.72 \pm 1.26(5)$ & $13.77 \pm 1.62(5)$ & $14.34 \pm 1.56(5)$ \\
Kidneys (ng/g) & $18.56 \pm 1.37(5)$ & $21.19 \pm 0.75(5)$ & $23.13 \pm 1.33(5)$ & $43.24 \pm 2.15^{\mathrm{a}}(5)$ \\
Brain (ng/g) & $17.62 \pm 1.71(5)$ & $19.77 \pm 0.96(5)$ & $15.03 \pm 1.10(5)$ & $17.02 \pm 0.94(5)$ \\
Olfactory bulb (ng/g) & $11.07 \pm 3.88(8)$ & $19.22 \pm 3.61(10)$ & $21.37 \pm 3.65(10)$ & $16.57 \pm 3.47(8)$ \\
Blood (ng/ml) & $6.93 \pm 0.61(8)$ & $5.75 \pm 0.73(10)$ & $6.41 \pm 0.65(10)$ & $6.86 \pm 0.89(8)$ \\
\hline
\end{tabular}

( ): number of animals, a: $p<0.01$, high group vs. other groups

the tidal volume appeared to be present in female rats, but was not statistically significant.

\section{Histopathologic Examination}

The only significant changes in histopathology occurred in lungs (Table 10 and 11), where there was minor focal inflammation with an inflammatory infiltrate of mixed cell type (lymphocyte/neutrophil/macrophage) were noted in both treated male and female rats. The increases were dose-dependent in female rats (Figure 9).

\section{Erythrocyte aggregation and kidney function test}

To evaluate changes in red blood cell aggregation or blood coagulation, erythrocyte aggregation, activated partial thromboplastin time (APPT), and prothrombin time (PT) were tested. No significant differences were found between the control and any treated animals in APPT or PT (Figure 10,A and 10B). There were no significant differences among dose groups in kidney function as measured based on the NAG and protein in the urine (Figure 11,A and 11B).

\section{Discussion}

To the best of our knowledge, this is the first subchronic 90-day inhalation study using gold nanoparticles to be reported in the peer-reviewed literature. An important aspect of this and predecessor studies from our laboratory is that the surface of the nanoparticles used for this exposure were not modified and not oxidized. There is one previous inhalation (intratracheal instillation) study which used $1.4 \mathrm{~nm}$ and $18 \mathrm{~nm}$ particles whose surface was ligated with $\mathrm{Ph}_{2} \mathrm{PC}_{6} \mathrm{H}_{4} \mathrm{SO}_{3} \mathrm{Na}$ [23] and one 15-day whole body inhalation study which used citrated $20 \mathrm{~nm}$ particles [24].

Interestingly, a recent publication [25] found that colloidal gold nanoparticles diluted in ultrapure water were well-dispersed, while agglomerates were formed when the diluent was phosphate buffered saline. In that study, rats were administered $50 \mathrm{~nm}$ and $250 \mathrm{~nm}$ gold particles by intratracheal injection. Despite differences in the degree of agglomeration due to the two diluents, no major differences in pulmonary and systemic toxicity markers were observed.

It is possible to calculate a deposited dose in the lung for this study. The deposited dose is calculated as follows:

Deposited dose $=($ minute volume $) \times($ number of minutes of exposure $) \times$ (concentration in air) $\times$ (fraction of the dose retained)

If the minute volume of male and female SpragueDawley rats in a subchronic study is 0.19 and $0.15 \mathrm{~L} / \mathrm{min}$, respectively [26] and the fraction of the dose deposited for particles less than $10 \mathrm{~nm}$ in diameter is 0.8 [27], then the deposited dose to the lung would be $0.14 \mu \mathrm{g}, 1.35 \mu \mathrm{g}$ and $71.1 \mu \mathrm{g}$ for the low, middle, and high dose males, respectively. For the low, middle, and high dose females, the deposited dose to the lung would be $0.11 \mu \mathrm{g}, 1.07 \mu \mathrm{g}$ and $56.2 \mu \mathrm{g}$, respectively. For comparison purposes and using the same assumptions, we can calculate deposited dose to the lung from our previously reported subchronic inhalation study of 18-19 nm silver particles [14]. The deposited dose to the lung for the silver study would be $170 \mu \mathrm{g}, 470 \mu \mathrm{g}$ and $1800 \mu \mathrm{g}$ for the low, middle, and high dose males, respectively. For the low, middle, and high

Table 9 Gold distribution in tissue of female rats after $\mathbf{9 0}$-day gold nanoparticle exposure.

\begin{tabular}{lcccc}
\hline GROUP: (mean \pm S.E) & Control & Low & Middle & High \\
\hline Lungs (ng/g) & $13.75 \pm 0.95(5)$ & $15.07 \pm 2.02(5)$ & $28.34 \pm 1.16(5)$ & $1960.02 \pm 92.80^{\mathrm{a}}(5)$ \\
Liver (ng/g) & $16.57 \pm 3.89(5)$ & $12.08 \pm 2.13(5)$ & $12.31 \pm 1.18(5)$ & $14.96 \pm 1.54(5)$ \\
Kidneys (ng/g) & $21.27 \pm 0.65(5)$ & $19.43 \pm 0.79(5)$ & $26.37 \pm 0.79(5)$ & $61.90 \pm 3.15^{\mathrm{a},{ }^{* *}}(5)$ \\
Brain (ng/g) & $19.13 \pm 2.31(5)$ & $18.42 \pm 1.07(5)$ & $17.87 \pm 0.86(5)$ & $22.15 \pm 0.75^{* * *}(5)$ \\
Olfactory bulb (ng/g) & $23.19 \pm 2.45(10)$ & $20.22 \pm 4.35(10)$ & $23.56 \pm 4.15(10)$ & $22.92 \pm 4.07(10)$ \\
Blood (ng/ml) & $6.66 \pm 0.78(10)$ & $6.92 \pm 1.08(10)$ & $5.10 \pm 0.58(10)$ & $8.98 \pm 1.25(10)$ \\
\hline
\end{tabular}

( ), number of animals; $a, p<0.01$ high group vs. other groups; ${ }^{* *}, p<0.01$ female rats vs. male rats in high group 


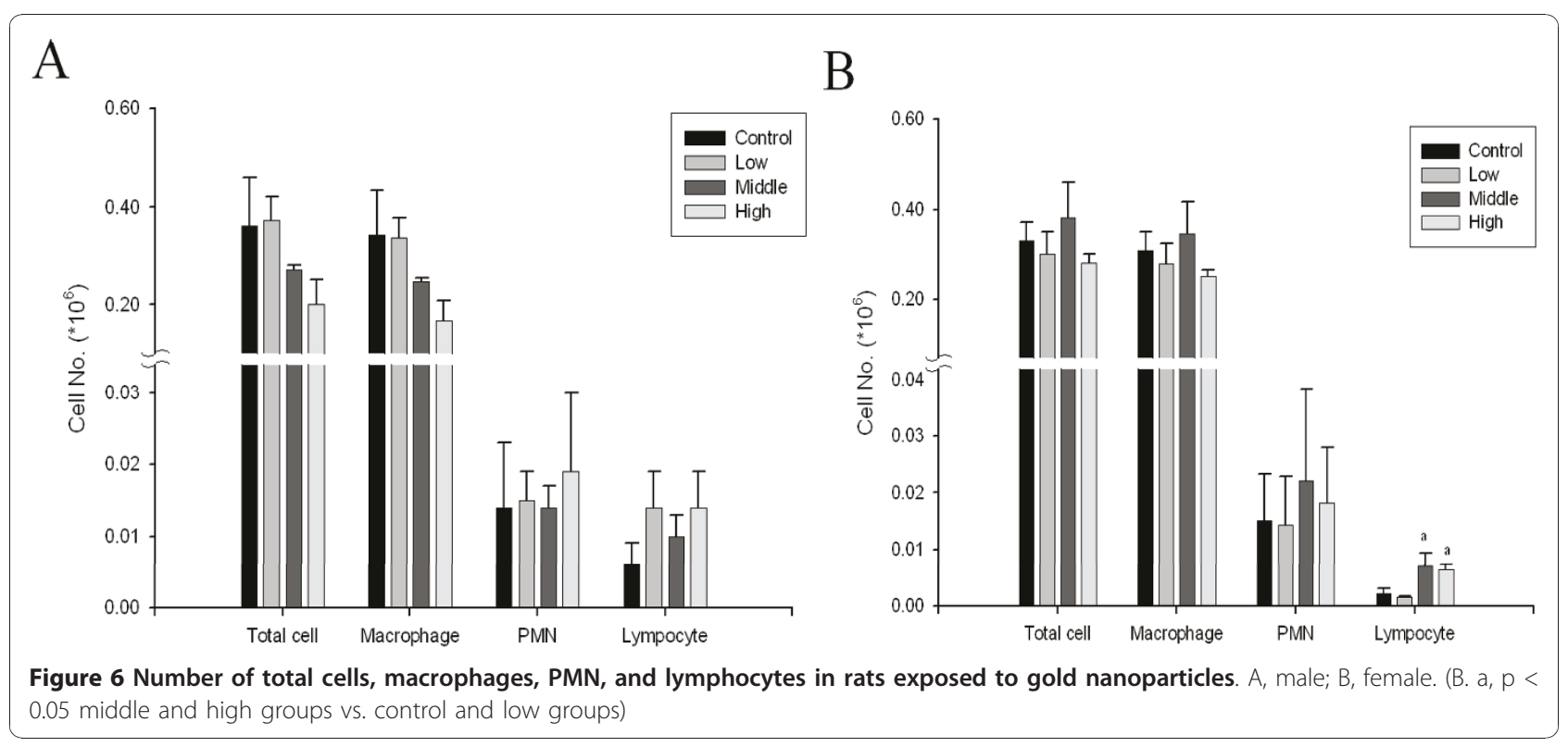

dose females, the deposited dose to the lung would be $140 \mu \mathrm{g}, 370 \mu \mathrm{g}$ and $1400 \mu \mathrm{g}$, respectively. Thus, the high deposited dose in our subchronic gold study is about $40 \%$ by weight of the low deposited dose in our subchronic silver study.

There is considerable uncertainty in the fraction of the dose retained for particles less than $10 \mathrm{~nm}$ in diameter as opposed to the fraction of the dose deposited as used in the calculation above. It is likely that the retained dose is significantly different than 0.8 due to such factors as translocation to other organs and species-specific deposition patterns. The reader is referred to more comprehensive discussions of retention of nanoparticles and deposition modeling in human and animal models [28-32].

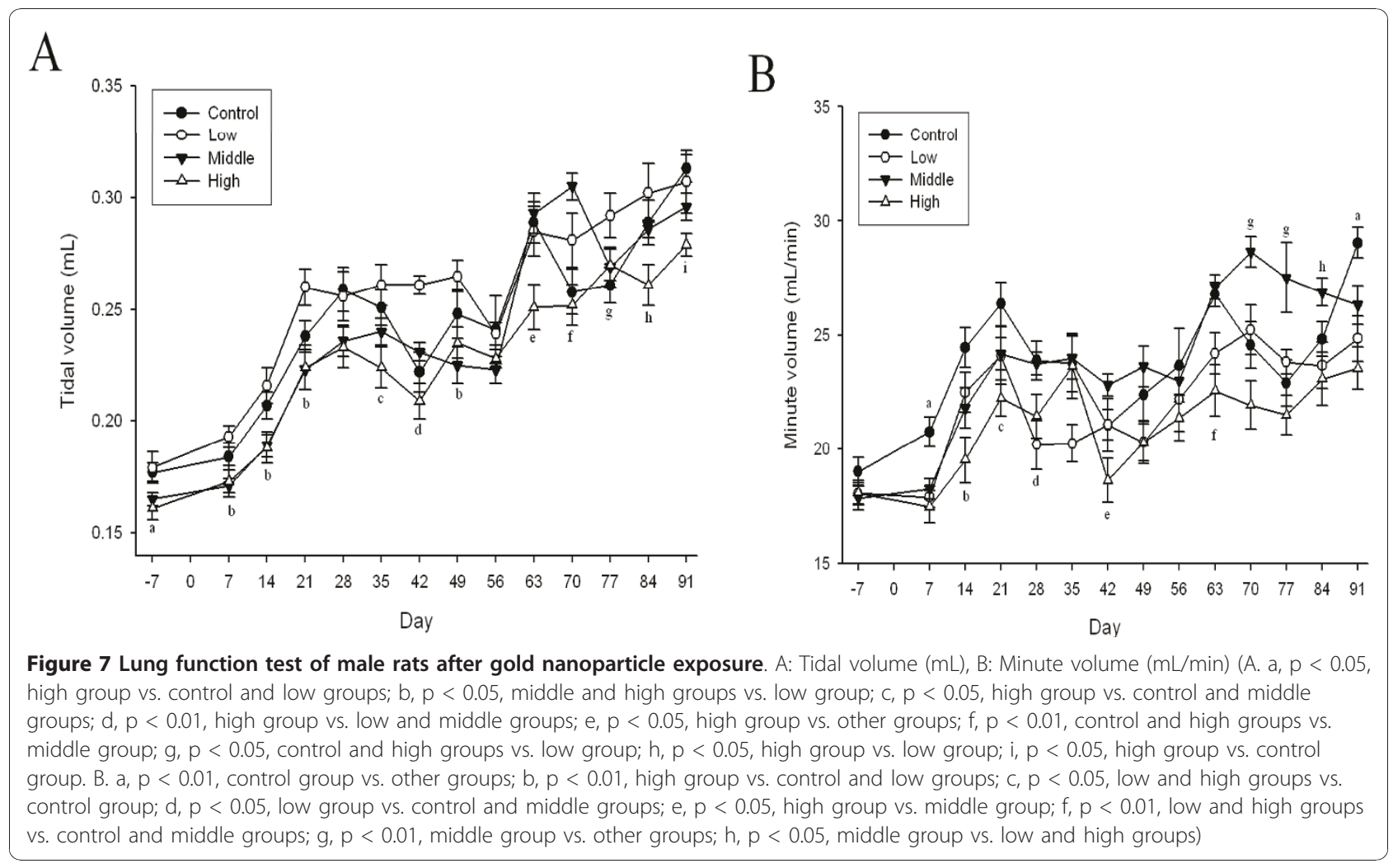




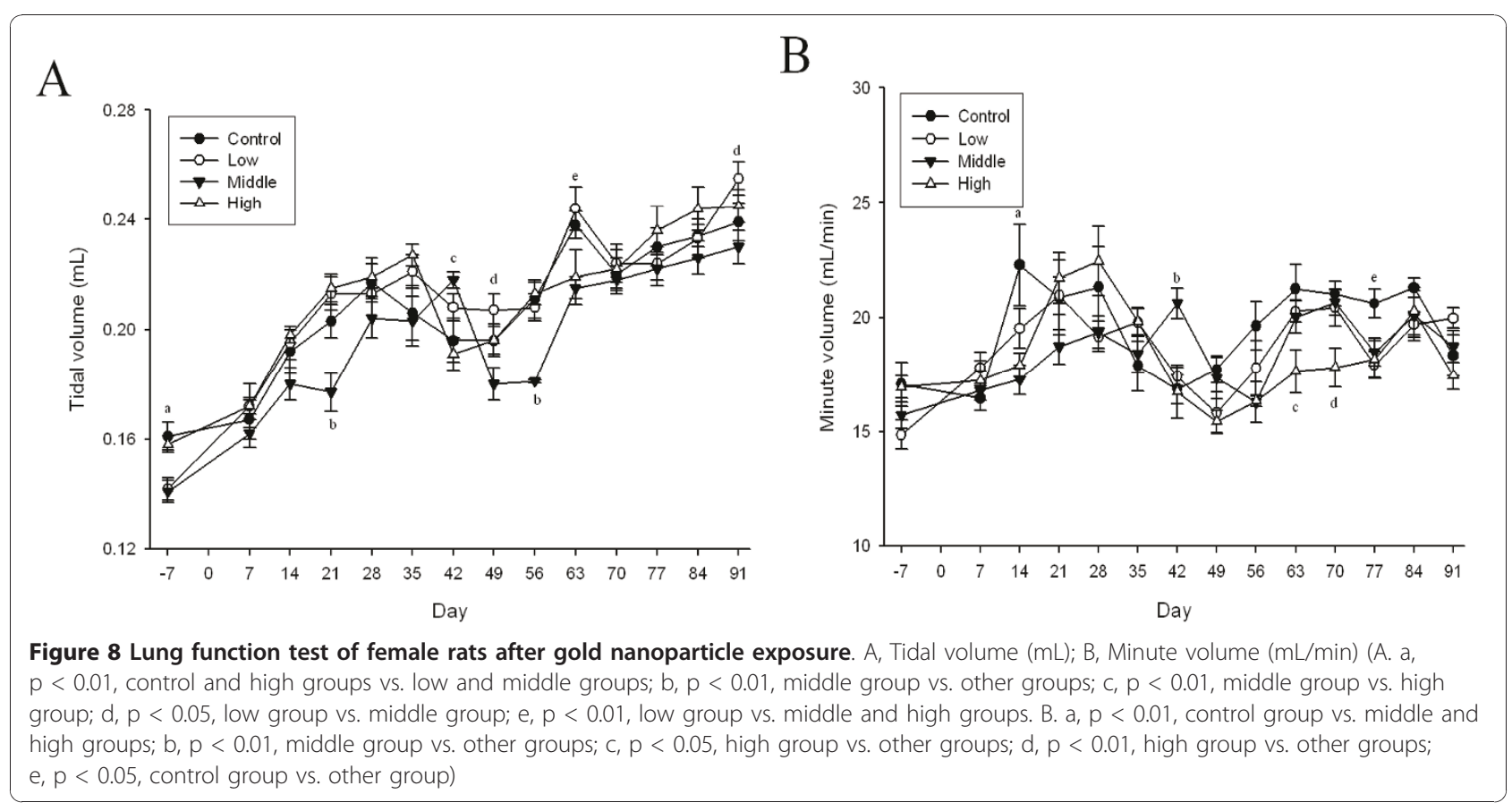

Whole body inhalation studies involve an additional component of exposure; that of ingestion since particles accumulate on the skin and fur. It is well known that rats clean skin and fur by licking, thus introducing particles to the gastrointestinal tract. For particles of gold larger than the nanometer range, it is generally assumed that the particles are not absorbed. Little is known about gastro-intestinal absorption of gold nanoparticles, but there are reports on the permeability of rat intestine to colloidal gold nanoparticles. Sonavane et al. [33] studied movements of 15,102 , and $198 \mathrm{~nm}$ colloidal gold particles across intestine in vitro. Fifteen nm particles were shown to cross the intestine more readily than 102 or $198 \mathrm{~nm}$ particles. The permeation of these particles through rat intestine was higher than rat skin. Hillyer et al. [34] studied absorption of 4, 10, 28, and 58 colloidal gold nanoparticles fed to mice. In general, smaller particles were absorbed more readily and corresponding tissue levels were higher. The applicability of this data to whole body inhalation is not apparent because of the colloidal nature of the tested particles [33,34]. Clearly, quantitative in vivo data on the absorption of unmodified gold nanoparticles in the rat would be of great value in determining the relative contribution of gastrointestinal absorption to the accumulation of gold from nanoparticles.

Table 10 Histopathologic observations for male rats.

\begin{tabular}{|c|c|c|c|c|c|c|c|c|c|c|c|c|}
\hline \multicolumn{5}{|c|}{ GROUP: } & \multicolumn{2}{|c|}{ Control } & \multicolumn{2}{|c|}{ Low } & \multicolumn{2}{|c|}{ Middle } & \multicolumn{2}{|c|}{ High } \\
\hline \multicolumn{5}{|c|}{ Number of Animals } & \multicolumn{2}{|c|}{8} & \multicolumn{2}{|c|}{10} & \multicolumn{2}{|c|}{10} & \multicolumn{2}{|c|}{8} \\
\hline & & & & & $\mathrm{N}$ & $\%$ & $\mathrm{~N}$ & $\%$ & $\mathrm{~N}$ & $\%$ & $\mathrm{~N}$ & $\%$ \\
\hline & No $m$ & oscopic findings & & & $8 / 8$ & 100 & $10 / 10$ & 100 & $6 / 10$ & 60 & $5 / 8$ & 62.5 \\
\hline & Abno & ality* & & & $0 / 8$ & 0 & $0 / 10$ & 0 & $4 / 10$ & 40 & $3 / 8$ & 37.5 \\
\hline \multirow[t]{5}{*}{ Liver } & & Inflammation & Focal & minimum & $0 / 8$ & 0 & $0 / 10$ & 0 & $0 / 10$ & 0 & $1 / 8$ & 12.5 \\
\hline & Sign & & Multifocal & minimum & $0 / 8$ & 0 & $0 / 10$ & 0 & $3 / 10$ & 30 & $0 / 8$ & 0 \\
\hline & & Necrosis & Focal & minimum & $0 / 8$ & 0 & $0 / 10$ & 0 & $0 / 10$ & 0 & $2 / 8$ & 25 \\
\hline & & Vacuolation & Hepatocellular & minimum & $0 / 8$ & 0 & $0 / 10$ & 0 & $1 / 10$ & 10 & $1 / 8$ & 12.5 \\
\hline & No $m$ & scopic findings & & & $7 / 8$ & 87.5 & $10 / 10$ & 100 & $10 / 10$ & 100 & $5 / 8$ & 62.5 \\
\hline \multirow[t]{3}{*}{ Lungs } & Abno & ality* & & & $1 / 8$ & 12.5 & $0 / 10$ & 0 & $0 / 10$ & 0 & $3 / 8$ & 37.5 \\
\hline & Sign & Inflammation* & Focal & minimum & $1 / 8$ & 12.5 & $0 / 10$ & 0 & $0 / 10$ & 0 & $3 / 8$ & 37.5 \\
\hline & & \multicolumn{3}{|c|}{ Osseous Metaplasia } & $0 / 8$ & 0 & $0 / 10$ & 0 & $0 / 10$ & 0 & $1 / 8$ & 12.5 \\
\hline
\end{tabular}

*, $\mathrm{p}<0.05$ compared with controls. Abnormality" refers to such changes as inflammation, vacuolization and necrosis upon histopathological examination. One abnormality is counted even if inflammation and necrosis are present simultaneously. 
Table 11 Histopathologic observations for female rats.

\begin{tabular}{|c|c|c|c|c|c|c|c|c|c|c|c|c|}
\hline \multicolumn{5}{|c|}{ GROUP: } & \multicolumn{2}{|c|}{ Control } & \multicolumn{2}{|c|}{ Low } & \multicolumn{2}{|c|}{ Middle } & \multicolumn{2}{|c|}{ High } \\
\hline \multicolumn{5}{|c|}{ Number of Animals } & \multicolumn{2}{|c|}{10} & \multicolumn{2}{|c|}{10} & \multicolumn{2}{|c|}{10} & \multicolumn{2}{|c|}{10} \\
\hline & & & & & $\mathrm{N}$ & $\%$ & $\mathrm{~N}$ & $\%$ & $\mathrm{~N}$ & $\%$ & $\mathrm{~N}$ & $\%$ \\
\hline & No $m$ & scopic findings & & & 9/10 & 90 & 9/10 & 90 & $8 / 10$ & 80 & $7 / 10$ & 70 \\
\hline & Abno & ality & & & $1 / 10$ & 10 & $1 / 10$ & 10 & $2 / 10$ & 20 & $3 / 10$ & 30 \\
\hline & & Inflammation & Focal & minimum & $0 / 10$ & 0 & $1 / 10$ & 10 & $0 / 10$ & 0 & $0 / 10$ & 0 \\
\hline \multirow[t]{5}{*}{ Liver } & & & & mild & $1 / 10$ & 10 & $0 / 10$ & 0 & $0 / 10$ & 0 & $0 / 10$ & 0 \\
\hline & Sign & Necrosis & Focal & minimum & $0 / 10$ & 0 & $0 / 10$ & 0 & $1 / 10$ & 10 & $1 / 10$ & 10 \\
\hline & & Vacuolization & Hepatocellular & minimum & $0 / 10$ & 0 & $0 / 10$ & 0 & $1 / 10$ & 10 & $2 / 10$ & 20 \\
\hline & & & & mild & $0 / 10$ & 0 & $0 / 10$ & 0 & $0 / 10$ & 0 & $1 / 10$ & 10 \\
\hline & No $m$ & scopic findings & & & $10 / 10$ & 100 & $10 / 10$ & 100 & $9 / 10$ & 90 & $3 / 10$ & 30 \\
\hline \multirow[t]{3}{*}{ Lungs } & Abno & ality** & & & $0 / 10$ & 0 & $0 / 10$ & 0 & $1 / 10$ & 10 & $7 / 10$ & 70 \\
\hline & Sign & Inflammation** & Focal & minimum & $0 / 10$ & 0 & $0 / 10$ & 0 & $1 / 10$ & 10 & $6 / 10$ & 60 \\
\hline & & & & mild & $0 / 10$ & 0 & $0 / 10$ & 0 & $0 / 10$ & 0 & $1 / 10$ & 10 \\
\hline
\end{tabular}

**, $\mathrm{p}<0.01$, compared with control. Abnormality" refers to such changes as inflammation, vacuolization and necrosis upon histopathological examination. One abnormality is counted even if inflammation and necrosis are present simultaneously

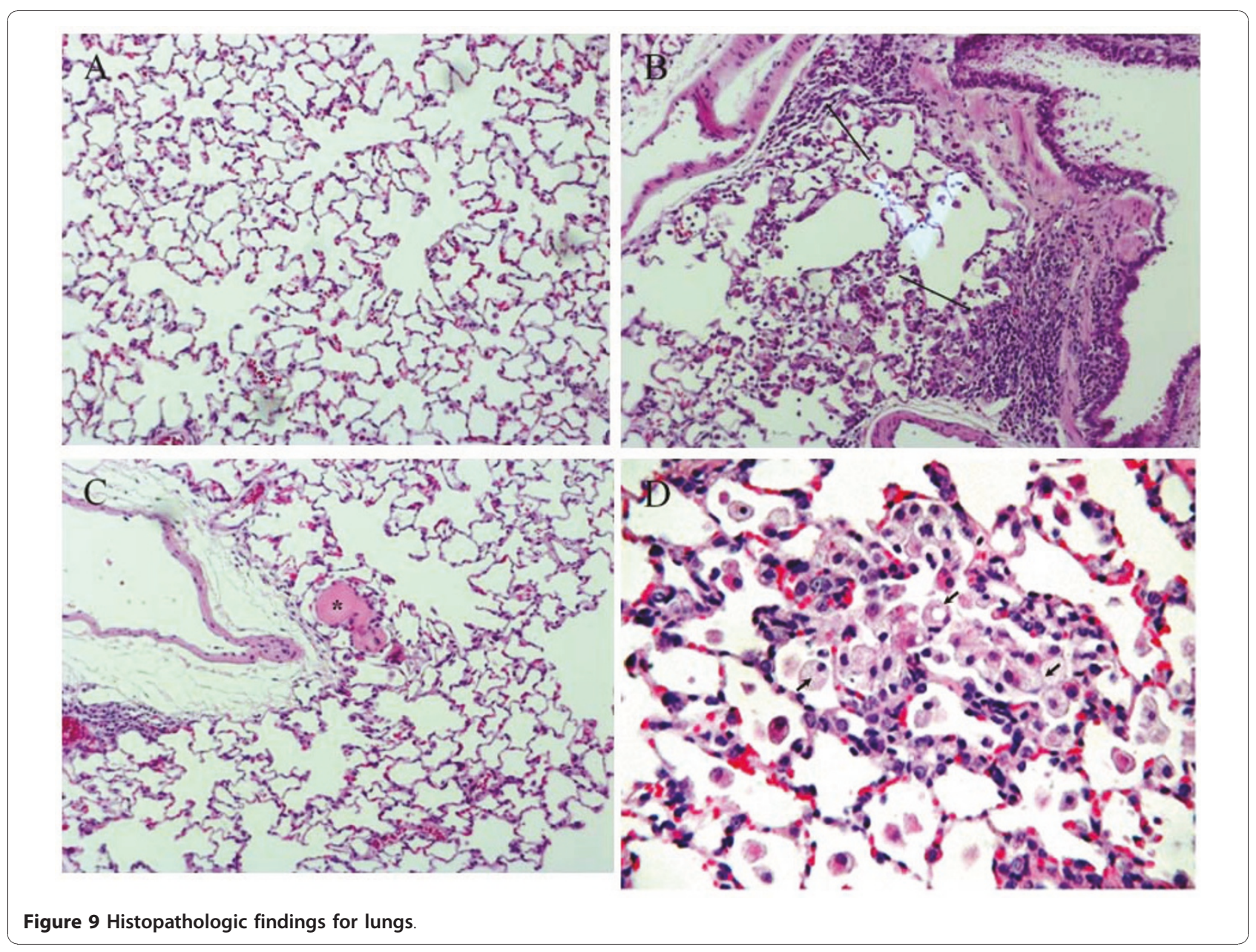




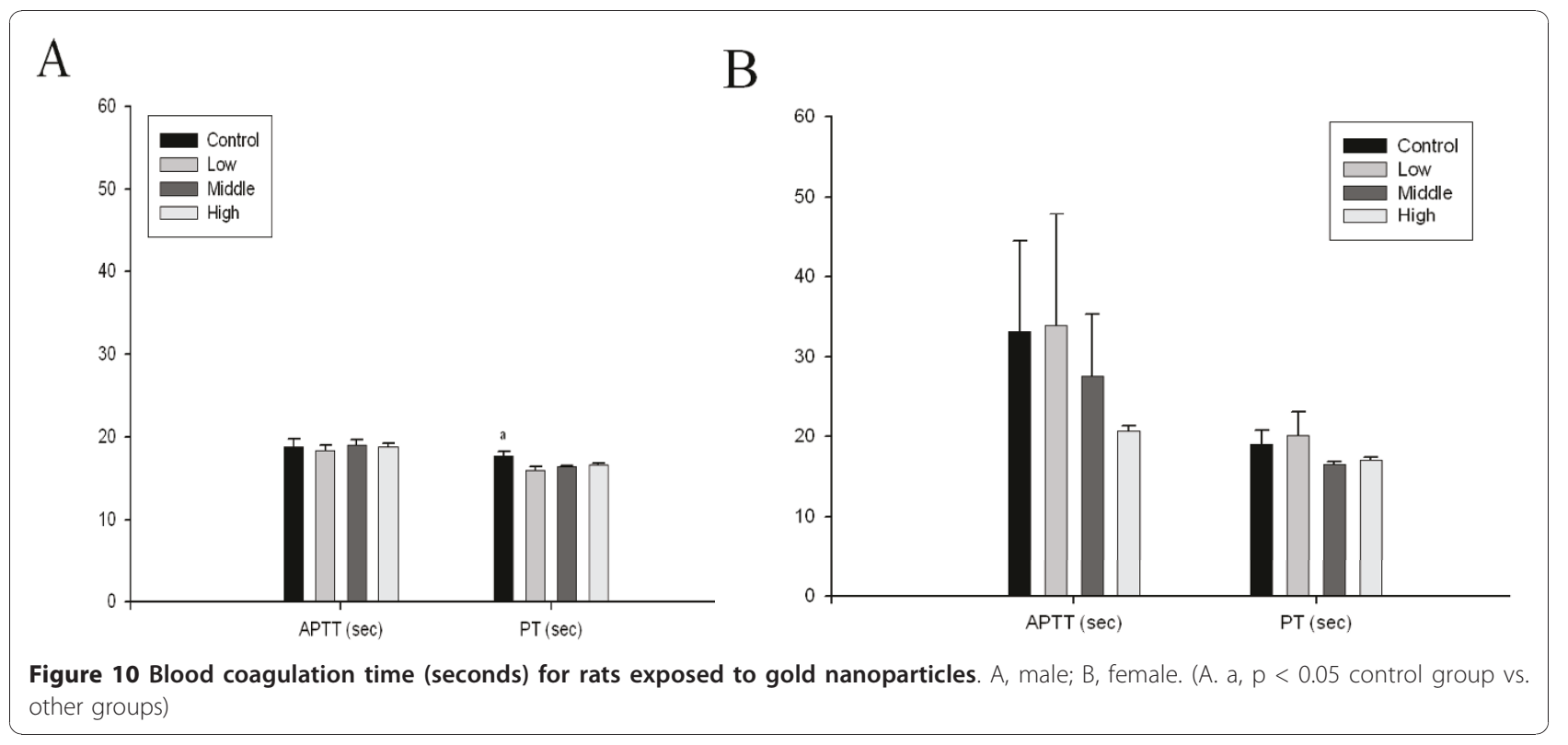

Tissue concentrations of gold from control rats have been reported in several studies $[24,35]$ and tend to be lower than found in our study (Table 8 and 9). Takenaka et al. [34] reported gold in control lungs to be 0.07 $\pm 0.003 \mathrm{ng} / \mathrm{g}$ and in control blood to be $0.1 \pm 0.07 \mathrm{ng} /$ $\mathrm{ml}$ (mean $\pm \mathrm{SD}$ ). Yu et al. [24] reported gold in control lungs to be $5.50 \pm 4.44 \mathrm{ng} / \mathrm{g}$ and in control blood to be $5.83 \pm 3.02 \mathrm{ng} / \mathrm{ml}$. Yu et al. [24] also reported gold in control olfactory bulb to be $4.44 \pm 4.43 \mathrm{ng} / \mathrm{g}$ and in control brain to be from $4.67 \pm 3.63$ to $20.27 \pm 15.79$ $\mathrm{ng} / \mathrm{g}$ (presumed to be mean $\pm \mathrm{SD}$ ) depending on the part of the brain analyzed. Because of the low levels of gold in tissues, the authors cannot rule out the possibility of contamination of internal tissues from skin or fur.
Mitigating that possibility is previous experience by the authors in whole-body inhalation studies involving nanoparticles. Nevertheless, the reader should consider the possibility for such contamination.

The results of this study indicated that the lungs were the major target tissue; pulmonary effects included a decrease in tidal and minute volume and the presence of mixed inflammatory cell infiltrates. Dose-related changes in tidal and minute volume tend to be obscured by changes over time which also occurred in control animals. In our experience, pulmonary function changes can reproduce poorly over time because they are dependent on so many variables. The decrease in pulmonary function following 90-days of gold nanoparticle
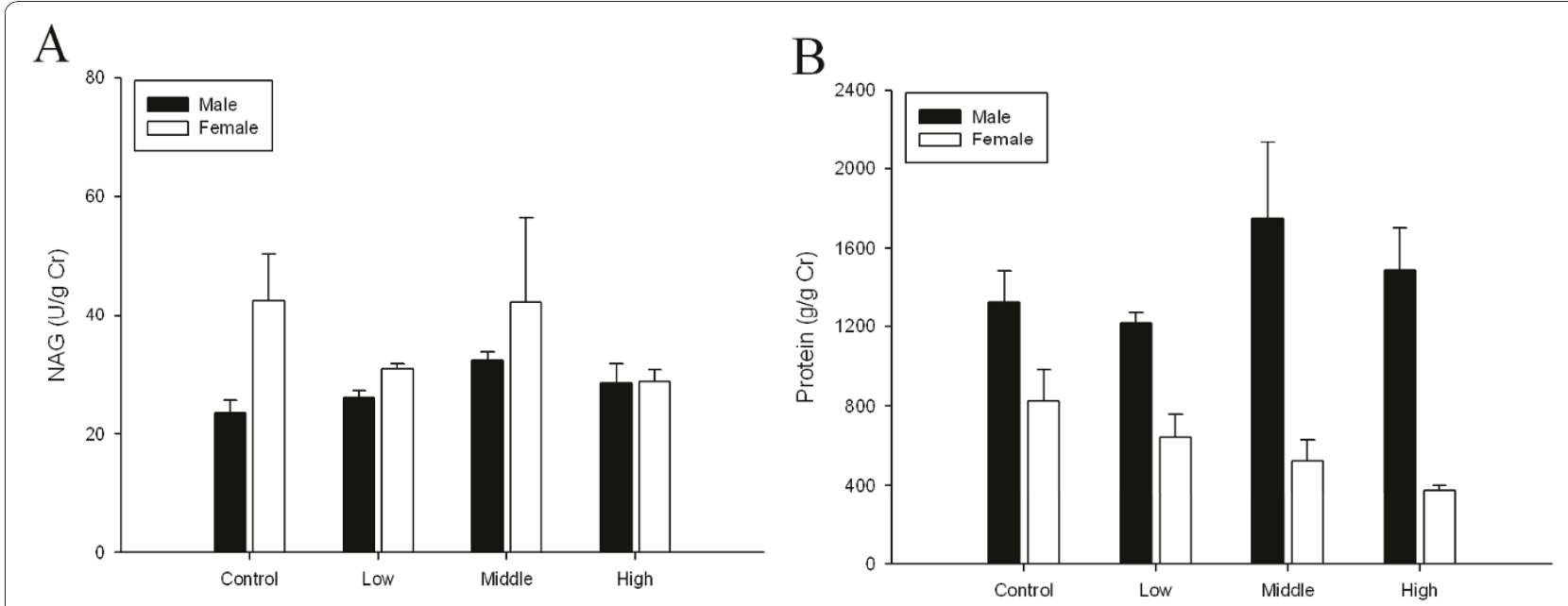

Figure 11 Kidney function test after 90-day gold nanoparticle exposure. A, NAG; B, Protein 
inhalation in the current study was similar, although lower, than that reported after 90-days of silver nanoparticle inhalation [14].

In the present study inhaled gold nanoparticles accumulated in a dose-dependent manner in lungs and kidneys of both male and female rats $(\mathrm{p}<0.01)$, but not in liver, blood, and olfactory bulb. This is in contrast to the study of $\mathrm{Yu}$ et al. [24] where rats are exposed to 20 $\mathrm{nm}$ gold nanoparticles at a concentration of $2 \times 10^{6} \%$ $\mathrm{cm}^{3}$ (mass concentration not reported) for 15 days by inhalation reported that the particles relocate from lungs to liver. Exposure in that study for 5 days resulted in a significant increase of gold in the lungs and olfactory bulb, as detected by ICP-MS; after 15 days of exposure, a significant accumulation of gold was detected in the lungs, esophagus, tongue, kidneys, aorta, spleen, septum, heart, and blood. Five or 15 days of inhalation exposure to gold nanoparticles resulted in a slight accumulation of gold in liver and a minimal increase the gold content of the olfactory bulb indicating a small but significant translocation from lung to blood after 15 days of exposure. Takenaka et al. [35] exposed rats to $16 \mathrm{~nm}$ gold particles at a concentration of $4 \times 10^{5} / \mathrm{cm}^{3}$ $\left(88 \mu \mathrm{g} / \mathrm{m}^{3}\right)$ for 6 hours and then serially sacrificed animals at $0,1,4$, and 7 days. These data also indicate a small but significant translocation from lung to blood.

The lack of significant increase in gold concentration in the olfactory bulb in our study is interesting with regard to findings in previously reported studies of shorter duration, particularly those of $\mathrm{Yu}$ et al. [24]. They showed a small but significant increase in the concentration of gold in the olfactory bulb and parts of the brain. The reason for the differences between our study and that of Yu et al. [24] are not apparent, but could be related to the surface composition of the nanoparticle itself, duration of exposure or the higher concentrations of gold in found in the olfactory bulbs of control animals.

The results of our 90-day study are not consistent with data from studies of nanoparticles of different composition and duration and stand in contrast to Elder et al. [36] using inhaled Mn oxides nanoparticles and Balasubramanian et al. [37] using intravenously injected gold nanoparticles. Elder et al. found that when rats inhaled manganese oxide particles (agglomerates measured 30 $\mathrm{nm}$ in diameter with primary particles of 3-8 nm) for 12 days, the particles accumulated in the olfactory bulb [36]. It is not clear if the differences between our study and that of Elder et al. are due to exposure duration, particle size, or solubility. Balasubramanian et al. [37] reported that a single intravenous injection of gold nanoparticles yielded a large amount of gold in the olfactory bulb (up to $72.2 \mathrm{ng} / \mathrm{g}$ ) two months after intravenous gold nanoparticle injection. A similar comparative distribution between intravenous injection and intratracheal instillation was also observed in a study by Semmler-Behnke et.al. [23]. Perhaps this concurrence of distribution is related to the fact that both intravenous injection and intratracheal installation deliver a large bolus of nanoparticles whereas normal inhalation delivers a much lower concentration of nanoparticles per unit time.

Throughout this study, male rats were larger than females (Tables 2 and 3, Figures 5A and 5B). There appeared to be no consistent differences between male and female in the organ content of gold and particularly lung indicating that differences in were not likely to have been due to a gender difference in deposition.

The higher accumulation of gold nanoparticles in the kidneys found in the present study was also previously observed in silver nanoparticle inhalation and oral exposure studies $[14,16]$, suggesting that the kidneys are the major accumulation site for metal nanoparticles whether the particles are ionized, like silver, or non-ionized, like gold. Gender-related accumulation of gold nanoparticles noted in this study was also observed for silver nanoparticles in the inhalation and oral exposure studies $[14,16]$. It therefore appears that at least over the range of average nanoparticle sizes of $5 \mathrm{~nm}$ (gold, inhalation), 15-20 $\mathrm{nm}$ (silver, inhalation), and up to $60 \mathrm{~nm}$ (silver, oral) there is a similar gender-related accumulation, and that there is a difference in the pattern of nanoparticle distribution between male and female kidneys. It is not apparent whether this difference is anatomically or hormonally-based.

In contrast to the 0-hypothesis for silver suggested by Wijnhoven et al. [38], in which silver toxicity mainly originates from silver ions generated from the surface of silver nanoparticles, the present results using gold nanoparticle inhalation provide a different view of nanoparticle toxicity and distribution. Gold nanoparticles (1.4 $\mathrm{nm}$ ) injected intravenously or administered by intratracheal instillation are excreted into the urine and not likely ionized in the body, they are translocated to tissues as particles rather than in an ionic form [23]. This implies that part or even a majority of the tissue distribution pattern of silver nanoparticles could be due to translocation in particulate form rather than ionic form and that most of the silver captured in kidneys is not in ionic form.

Currently, there are no occupational exposure standards for gold dust, fumes, or nanoparticles other than general particulate standards such as the "particles not otherwise regulated" standard of the US OSHA permissible exposure limit (PEL) of $5 \mathrm{mg} / \mathrm{m}^{3}$ for respirable particles. Changes observed in lung histopathology and function in high-dose animals appear in and of themselves to be minor. In the previously reported study on 
silver nanoparticles, similar changes were noted [14] and the authors interpreted them to be transient and not sufficient to establish an effects level. It appears that our original interpretation was not correct since when rats from a recent 12 week exposure study similar to Sung et al. [15] were allowed to "recover," decreases in tidal and minute volumes from the middle and high dose groups have persisted up to 12 weeks post-exposure (unpublished data). In light of similar changes in this study, it appears that the highest concentration $(20 \mu \mathrm{g} /$ $\left.\mathrm{m}^{3}\right)$ is a LOAEL and the middle concentration $(0.38 \mu \mathrm{g} /$ $\mathrm{m}^{3}$ ) as a NOAEL.

\section{Acknowledgements}

Funding contributed by the "Nanosafety project" from the National Institute of Toxicological Research, Korean Food and Drug Administration.

\section{Author details \\ ${ }^{1}$ Toxicity Evaluation Team, Korea Conformity Laboratories, Incheon, Korea. ${ }^{2}$ DMC R\&D Center, Samsung Electronics Co. Ltd., Suwon, Korea. ${ }^{3}$ College of Medicine, Chung-Ang University, Seoul, Korea. ${ }^{4}$ R\&D Center, HCT Co. Icheon, Korea. ${ }^{5}$ National Institute of Food and Drug Safety Research, Korea Food \& Drug Administration, Ochang, Korea. ${ }^{6}$ Center for Occupational Toxicology, KOSHA, Daejeon, Korea. ${ }^{7}$ College of Medicine, Kosin University, Busan, Korea. ${ }^{8}$ Veritox, Inc. Seattle, USA. ${ }^{9}$ Toxicologcial Research Center, Hoseo University, Asan, Korea.}

\section{Authors' contributions}

IJY headed the study and performed the pathology together with MYS, YHC, $\mathrm{BSH}, \mathrm{JHJ}$ and HKC performed the pathology peer-review. JHJ, JUY, and KSJ generated and monitored gold nanoparticles in the inhalation chamber. IJY drafted the manuscript with JHS and BK. JHS headed all animal treatments with MYS, KSS and HRR. JDP, DWK and JHL contributed tissues distribution study. IJY and BK conceived and designed the study. All authors reviewed and interpreted data and read and approved the final manuscript

\section{Competing interests}

The authors declare that they have no competing interests.

Received: 3 March 2010 Accepted: 14 May 2011 Published: 14 May 2011

\section{References}

1. Merchant B: Gold, the noble metal and the paradoxes of its toxicology. Biologicals 1998, 26(1):49-59.

2. Forte G, Petrucci F, Bocca B: Metal allergens of growing significance: epidemiology, immunotoxicology, strategies for testing and prevention. Inflamm Allergy Drug Targets 2008, 7(3):145-62.

3. Eisler R: Mammalian sensitivity to elemental gold $\left(\mathrm{Au}^{\circ}\right)$. Biol Trace Elem Res 2004, 100(1):1-18.

4. Roenigk HR Jr, Handel D: Gold vasculitis. Arch Dermatol 1974, 109(2):253-255.

5. Comaish S: A case of contact hypersensitivity to metallic gold. Arch Dermatol 1969, 99(6):720-3.

6. Brown DH, Smith WE, Fox P, Sturrock RD: The reactions of gold (0) with amino acids and the significance of these reactions in the biochemistry of gold. Inorg Chimica Acta 1982, 67:27-30.

7. Woodrow Wilson International Center for Scholars: A nanotechnology consumer products inventory [http://www.nanotechproject.org/ consumerproducts], Accessed on 1/25, 2010.

8. National Toxicology Program (NTP), NTP Research Concept: Nanoscale Gold. [http://ntp.niehs.nih.gov/ntp/Noms/Final_ResConcept/ Nanoscale_Gold.pdf], accessed on 1/25, 2010.

9. Organization for Economic Cooperation and Development (OECD): OECD guidelines for the testing of chemicals. Test guideline 413. Subchronic inhalation toxicity: 90 day study Paris:OECD; 1995.
10. Ji JH, Jung JH, Yu IJ, Kim SS: Long-term stability characteristics of metal nanoparticle generator using small ceramic heater for inhalation toxicity studies. Inhalation Toxicology 2007, 19(9):745-51.

11. Ji JH, Jung JH, Kim SS, Yoon JU, Park JD, Choi BS, Chung YH, Kwon $\mathbb{H}_{\text {, }}$ Jeong J, Han BS, Shin JH, Sung JH, Song KS, Yu IJ: Twenty-eight-day inhalation toxicity study of silver nanoparticles in Sprague-Dawley Rats, Inhalation Toxicology. 2007, 19(10):857-871.

12. Jung JH, Oh HC, Noh HS, Ji JH, Kim SS: Metal nanoparticle generation using a small ceramic heater with a local heating area. J Aerosol Sci 2006, 37:1662-1670.

13. Jung JH, Oh HC, Ji JH, Kim SS: In-situ gold nanoparticle generation using a small-sized ceramic heater with a local heating area. Materials Science Forum 2007, 544-545:1001-1004.

14. Sung JH, Ji JH, Park JD, Yoon JU, Kim DS, Jeon KS, Song MY, Jeong J, Han BS, Han JH, Chung YH, Chang HK, Lee JH, Cho MH, Kelman BJ, Yu IJ: Subchronic inhalation toxicity of silver nanoparticles, Toxicol Sci. 2009, 108(2):452-61.

15. Sung JH, Ji JH, Yun JU, Kim DS, Song MY, Jeong J, Han BS, Han JH, Chung YH, Kim J, Kim TS, Chang HK, Lee EJ, Lee JH, Yu IJ: Lung function changes in Sprague-Dawley rats after prolonged inhalation exposure to silver nanoparticles, Inhalation Toxicology. 2008, 20(6):567-74.

16. Kim YS, Kim JS, Cho HS, Rha DS, Kim JM, Park JD, Choi BS, Lim R, Chang HK, Chung YH, Kwon IH, Jeong J, Han BS, Yu IJ: Twenty eight-day oral toxicity, genotoxicity, and gender-related tissue distribution of silver nanoparticles in Sprague-Dawley Rats, Inhalation Toxicology. 2008, 20(6):575-83.

17. Antonini JM, Krishna-Murthy GG, Rogers RA, Albert R, Ulrich GD, Brain JD: Pneumotoxicity and pulmonary clearance of different welding fumes after intratracheal instillation in the rat. Toxicol Appl Pharmacol 1996, 114:188-199.

18. Antonini JM, Krishna-Murthy GG, Brain JD: Responses to welding fume: Lung injury inflammation and release of tumor necrosis factor-alpha and interleukin-1 beta. Exp Lung Res 1997, 23:205-227.

19. Lemaire I, Ouellet $\mathrm{S}$ : Distinctive profile of alveolar macrophage derived cytokine release induced by fibrogenic and nonfibrogenic mineral dusts. J Toxicol Environ Health 1996, 47:465-478.

20. Sung JH, Choi BG, Maeng SH, Kim SJ, Chung YH, Han JH, Song KS, Lee YH, Cho YB, Cho MH, Kim KJ, Hyun JS, Yu IJ: Recovery from welding fume induced lung fibrosis and pulmonary function changes in Sprague Dawley rats. Tox Sci 2004, 82:608-613.

21. Adamson IYR, Prieditis H, Hedgecock C: Pulmonry response of mice to fiberglass: Cytokinetic and biochemical studies. $J$ Toxicol Environ Health 1995, 46:411-424.

22. NIOSH, NIOSH manual of analytical methods: Method No. 7300, 7604. National Institute for Occupational Safety and Health; Cincinnati. NIOSH: 1999.

23. Semmler-Behnke M, Kreyling WG, Lipka J, Fertsch S, Wenk A, Takenaka S, Schmid G, Brandau W: Biodistribution of 1.4- and 18-nm gold particles in Rats, Small. 2008, 4(12):2108-2111.

24. Yu LE, Yung LYK, Ong CN, Tan YL, Balasubramaniam KS, Hartono D, Shui G, Wenk MR, Ong WY: Translocation and effects of gold nanoparticles after inhalation exposure in rats, Nanotoxicology. 2007, 1(3):235-242.

25. Gosens I, Post JA, de la Fonteyne L, Jansen E, Geus J, Cassee F, Jong W: Impact of agglomeration state of nano- and submicron sized gold particles on pulmonary inflammation, Particle and Fibre Toxicology. 2010, 7:37.

26. Whalen J, Foureman $G$, Vandenberg J: Inhalation risk assessment at the Environmental Protection Agency, in Inhalation Toxicology.Edited by: Salem H, Katz S. Taylor and Francis Group, 6000 Broken Sound Parkway, Boca Raton, Fl; 2006:3-37.

27. Kim CS: Method of calculating lung delivery and deposition of aerosol particles. Respir Care 2000, 45(6):695-710.

28. Aillon KL, Xie Y, El-Gendy N, Berkland CJ, Forrest ML: Effects of nanomaterial physiochemical properties on in vivo toxicity, Advanced Drug Delivery Reviews. 2009, 61:457-466.

29. Choi Jl, Kim CS: Mathematical analysis of particle deposition in human lungs: an improved single path transport model, Inhalation Toxicology. 2007, 19:925-939.

30. Kreyling WG, Semmler-Behnke M, Möller W: Dosimetry and toxicology of ultrafine particles, Journal of Aerosol Medicine. 2004, 17(2):140-152. 
31. Kreyling WG, Semmler-Behnke M, Möller W: Ultrafine particle-lung interactions: does size matter? Journal of Aerosol Medicine 2006, 19(1):74-83.

32. Varghese SK, Ganganna S: Particle deposition in human respiratory system: deposition of concentrated hydroscopic aerosols, Inhalation Toxicology. 2009, 21(7):619-630.

33. Sonavane G, Tomoda K, Sano A, Ohshima H, Terada H, Makino K: In vitro permeation of gold nanoparticles through rat skin and rat intestine: effect of particle size, Colloids and Surfaces B:Biointerfaces. 2009, 65:1-10.

34. Hillyer JF, Albrecht RM: Gastrointestinal persorption and tissue distribution of differently sized colloidal gold nanoparticles, Journal of Pharmaceutical Sciences. 2001, 90(12):1927-1935.

35. Takenaka S, Karg E, Kreyling WG, Lentner B, Möller W, Behnke-Semmler M, Jennen I, Walch A, Michalke B, Schramel P, Heyder J, Schulz H: Distribution pattern of inhaled ultrafine gold particles in the rat lung, Inhalation Toxicology. 2006, 18:733-740.

36. Elder A, Gelein R, Silva V, Feikert T, Opanashuk L, Carter J, Potter R, Maynard A, Ito Y, Finkelstein J, Oberdörster G: Translocation of inhaled ultrafine manganese oxide particles to the central nervous system, Environment Health Perspectives. 2006, 114(8):1172-1178.

37. Balasubramanian SK, Jittiwat J, Manikandan J, Ong CN, Yu LE, Ong WY: Biodistribution of gold nanoparticles and gene expression changes in the liver and spleen after intravenous administration in rats. Biomaterials 2010, 31(8):2034-42.

38. Wijnhoven SWP, Peijnenburg WJGM, Herberts CA, Hagens WI, Oomen AG, Heugens EHW, Roszek B, Bisschops J, Gosens I, De Meent BR, Dekkers S, De Jong WH, Van MV, Sips Jijverden AJAM, Geertsma RE: Nano-silver a review of available data and knowledge gaps in human and environmental risk assessment, Nanotoxicology. 2009, 3(2):109-138.

doi:10.1186/1743-8977-8-16

Cite this article as: Sung et al: Subchronic inhalation toxicity of gold nanoparticles. Particle and Fibre Toxicology 2011 8:16.

\section{Submit your next manuscript to BioMed Central and take full advantage of:}

- Convenient online submission

- Thorough peer review

- No space constraints or color figure charges

- Immediate publication on acceptance

- Inclusion in PubMed, CAS, Scopus and Google Scholar

- Research which is freely available for redistribution

Submit your manuscript at www.biomedcentral.com/submit
C Biomed Central 